\title{
18 Sco: A SOLAR TWIN RICH IN REFRACTORY AND NEUTRON-CAPTURE ELEMENTS. IMPLICATIONS FOR CHEMICAL TAGGING*
}

\author{
Jorge Meléndez ${ }^{1}$, Iván Ramírez ${ }^{2}$, Amanda I. Karakas ${ }^{3}$, David Yong ${ }^{3}$, TalaWanda R. Monroe ${ }^{1}$, Megan Bedell $^{4}$, \\ Maria BergemanN ${ }^{5}$, Martin Asplund ${ }^{3}$, Marcelo Tucci Maia ${ }^{1}$, Jacob Bean ${ }^{4}$, José-Dias do Nascimento Jr. ${ }^{6,7}$,

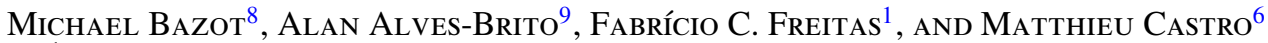 \\ ${ }^{1}$ Departamento de Astronomia do IAG/USP, Universidade de São Paulo, Rua do Matão 1226, 05508-900 \\ São Paulo, SP, Brazil; jorge.melendez@iag.usp.br \\ ${ }^{2}$ McDonald Observatory and Department of Astronomy, University of Texas at Austin, USA \\ ${ }^{3}$ Research School of Astronomy and Astrophysics, The Australian National University, Cotter Road, Weston, ACT 2611, Australia \\ ${ }^{4}$ Department of Astronomy and Astrophysics, University of Chicago, 5640 South Ellis Avenue, Chicago, IL 60637, USA \\ ${ }^{5}$ Institute of Astronomy, University of Cambridge, Madingley Road, CB3 OHA, Cambridge, UK \\ ${ }^{6}$ Departamento de Física Teórica e Experimental, Universidade Federal do Rio Grande do Norte, 59072-970 Natal, RN, Brazil \\ ${ }^{7}$ Harvard-Smithsonian Center for Astrophysics, Cambridge, MA 02138, USA \\ ${ }^{8}$ Centro de Astrofísica da Universidade do Porto, Rua das Estrelas, 4150-762 Porto, Portugal \\ ${ }^{9}$ Instituto de Fisica, Universidade Federal do Rio Grande do Sul, Av. Bento Goncalves 9500, Porto Alegre, RS, Brazil \\ Received 2014 May 3; accepted 2014 June 19; published 2014 July 21
}

\begin{abstract}
We study with unprecedented detail the chemical composition and stellar parameters of the solar twin 18 Sco in a strictly differential sense relative to the Sun. Our study is mainly based on high-resolution $(R \sim 110,000)$, high signal-to-noise ratio (800-1,000) Very Large Telescope UVES spectra, which allow us to achieve a precision of about 0.005 dex in differential abundances. The effective temperature and surface gravity of $18 \mathrm{Sco}$ are $T_{\text {eff }}=$ $5823 \pm 6 \mathrm{~K}$ and $\log g=4.45 \pm 0.02$ dex, i.e., $18 \mathrm{Sco}$ is $46 \pm 6 \mathrm{~K}$ hotter than the Sun and $\log g$ is $0.01 \pm 0.02 \mathrm{dex}$ higher. Its metallicity is $[\mathrm{Fe} / \mathrm{H}]=0.054 \pm 0.005 \mathrm{dex}$, and its microturbulence velocity is $+0.02 \pm 0.01 \mathrm{~km} \mathrm{~s}$ higher than solar. Our precise stellar parameters and differential isochrone analysis show that 18 Sco has a mass of $1.04 \pm 0.02 M_{\odot}$ and that it is $\sim 1.6$ Gyr younger than the Sun. We use precise High Accuracy Radial velocity Planet Searcher (HARPS) radial velocities to search for planets, but none are detected. The chemical abundance pattern of $18 \mathrm{Sco}$ displays a clear trend with condensation temperature, thus showing higher abundances of refractories in 18 Sco than in the Sun. Intriguingly, there are enhancements in the neutron-capture elements relative to the Sun. Despite the small element-to-element abundance differences among nearby n-capture elements $(\sim 0.02$ dex $)$, we successfully reproduce the $r$-process pattern in the Solar System. This is independent evidence for the universality of the $r$ process. Our results have important implications for chemical tagging in our Galaxy and nucleosynthesis in general.
\end{abstract}

Key words: stars: abundances - stars: AGB and post-AGB - stars: fundamental parameters - Sun: abundances

Online-only material: color figures, machine-readable table

\section{INTRODUCTION}

Solar twins are stars nearly indistinguishable from the Sun (Cayrel de Strobel 1996). The star 18 Sco was first identified as a solar twin by Porto de Mello \& da Silva (1997). This star has great importance because it is the brightest $(V=5.51$; Ramírez et al. 2012) and closest (13.9 pc) solar twin (Porto de Mello \& da Silva 1997; Soubiran \& Triaud 2004; Takeda et al. 2007; Datson et al. 2012, 2014; Porto de Mello et al. 2014); thus it can be studied through a variety of techniques. Moreover, 18 Sco has a declination of $-8^{\circ}$ and hence is observable from both the Northern and Southern Hemispheres.

Besides the many recent high-resolution chemical abundance studies (e.g., Luck \& Heiter 2005; Meléndez \& Ramírez 2007; Neves et al. 2009; Ramírez et al. 2009a; Takeda \& Tajitsu 2009; González Hernández et al. 2010; da Silva et al. 2012; Monroe et al. 2013), 18 Sco has been observed for chromospheric activity (e.g., Hall et al. 2007), magnetic fields (Petit et al. 2008), debris disks (e.g., Trilling et al. 2008), companions through

\footnotetext{
* Based on observations obtained at the European Southern Observatory (ESO) Very Large Telescope (VLT) at Paranal Observatory; the $3.6 \mathrm{~m}$ telescope at La Silla Observatory, Chile (observing programs 083.D-0871 and 188.C-0265); and at the W.M. Keck Observatory, which is operated jointly by Caltech, the University of California, and NASA.
}

high-resolution imaging (e.g., Tanner et al. 2010), granulation (Ramírez et al. 2009b), seismology (Bazot et al. 2011, 2012), and interferometry (Bazot et al. 2011; Boyajian et al. 2012). In addition, different techniques can be combined to obtain further insights on the fundamental properties of this solar twin (Li et al. 2012).

Most previous abundance studies on 18 Sco report a somewhat enhanced (about 10-15\%) iron abundance and a Li content about three to four times higher than solar, but otherwise approximately solar abundance ratios for other elements (e.g., Porto de Mello \& da Silva 1997; Meléndez \& Ramírez 2007; Takeda $\&$ Tajitsu 2009), except for some recent high-precision studies on 18 Sco (e.g., Meléndez et al. 2009; Ramírez et al. 2009a; Monroe et al. 2013), which show a clear trend with condensation temperature. The situation regarding the heavy elements is less clear, with Porto de Mello \& da Silva (1997) reporting a slight excess in the elements heavier than $\mathrm{Sr}$, but the recent study by Mishenina et al. (2013a) finds approximately solar ratios for the neutron-capture elements in 18 Sco. Instead, da Silva et al. (2012) found a clear enhancement in Sr, Ba, Nd, and Sm but solar ratios for $\mathrm{Y}$ and $\mathrm{Ce}$. To further complicate the situation, González Hernández et al. (2010) found a solar ratio for Nd (an element that was found enhanced by da Silva et al. (2012)), but $\mathrm{Eu}$ and $\mathrm{Zr}$ showed the largest enrichment. The worst cases are 
$\mathrm{Zr}$ and $\mathrm{Nd}$, with a spread of 0.15 dex and 0.14 dex, respectively, and individual values of $[\mathrm{Zr} / \mathrm{Fe}]=-0.05,+0.06,+0.10$, and $[\mathrm{Nd} / \mathrm{Fe}]=-0.01,0.13,0.00$, according to Mishenina et al. (2013a), da Silva et al. (2012), and González Hernández et al. (2010), respectively.

In order to better understand the likely chemical differences between 18 Sco and the Sun and to clarify the situation regarding the heavy elements $(Z>30)$, we perform a highly precise abundance analysis of 18 Sco for 38 chemical elements, thus making it the most complete and precise abundance study to date on the chemical composition of $18 \mathrm{Sco}$. In addition, our precise stellar parameters will be used in a forthcoming paper to better constrain the fundamental properties of 18 Sco in synergy with other techniques such as asteroseismology (M. Bazot et al., in preparation).

Our work is also relevant regarding "chemical tagging" (Freeman \& Bland-Hawthorn 2002), which aims to reconstruct the buildup of our Galaxy by identifying stars with a common origin. Although the dynamical information about their origin could have been lost, the chemical information should be preserved. In this context, the disentangling of the complex abundance pattern of 18 Sco can help us to assess which elements should be targeted for chemical tagging.

The analysis is mainly based on UV-optical spectra acquired with the Ultraviolet and Visual Echelle Spectrograph (UVES) at the Very Large Telescope (VLT) and complemented with optical spectra taken with the High-Resolution Echelle Spectrometer (HIRES) at Keck.

\section{OBSERVATIONS}

In order to cover a wide spectral range, we observed 18 Sco and the Sun (using solar reflected light from asteroids) in different spectrograph configurations. Both $18 \mathrm{Sco}$ and the reference solar spectrum were acquired in the same observing runs and using identical setups. We obtained visitor mode observations with the UVES spectrograph at the VLT (2009 August 30) and with the HIRES spectrograph at Keck (2005 June 16), covering with both data sets the UV/optical/near-IR spectrum from 306 to $1,020 \mathrm{~nm}$.

The UVES observations were taken in dichroic mode, obtaining thus simultaneous UV (blue arm) plus optical (red arm) coverage with the standard settings $346 \mathrm{~nm}+580 \mathrm{~nm}$, and another set of observations with the standard $346 \mathrm{~nm}$ setting plus a nonstandard setting centered at $830 \mathrm{~nm}$. With this configuration we achieved a high signal-to-noise ratio $(\mathrm{S} / \mathrm{N})$ in the UV, as the $346 \mathrm{~nm}$ setting (306-387 nm) was covered in both setups. The $580 \mathrm{~nm}$ standard setting covered the optical (480-682 $\mathrm{nm})$ region, and our $830 \mathrm{~nm}$ setting included the red region $(642-1020 \mathrm{~nm})$. Notice that our nonstandard setting at $830 \mathrm{~nm}$ was chosen to overlap the $580 \mathrm{~nm}$ setting in the 642-682 nm interval, so a higher $\mathrm{S} / \mathrm{N}$ was achieved around $670 \mathrm{~nm}$ in order to measure lithium with extremely high precision (e.g., Monroe et al. 2013).

The bulk of the analysis is based on the UVES optical spectra obtained in the red arm covering the 480-1,020 $\mathrm{nm}$ region. The observations with the red arm were obtained using the 0.3 arcsec slit, achieving a high resolving power $(R=110,000)$ and a very high $\mathrm{S} / \mathrm{N}$ (typical $\mathrm{S} / \mathrm{N} \sim 800 \mathrm{pixel}^{-1}$, and around the $\mathrm{Li}$ feature $\mathrm{S} / \mathrm{N} \sim 1000$ ). Some elements showing lines in the UV were studied with the UVES blue arm using the $346 \mathrm{~nm}$ setup (306-387 nm) with a slit of 0.6 arcsec, resulting in highresolution $(R=65,000)$, high $\mathrm{S} / \mathrm{N}(\sim 600$ at $350 \mathrm{~nm})$ spectra. The asteroid Juno was employed to obtain our reference solar spectrum for the UVES observations, and similar $\mathrm{S} / \mathrm{Ns}$ were achieved both for 18 Sco and Juno.

The spectral regions $387-480 \mathrm{~nm}$ and $577-585 \mathrm{~nm}$ are missing in our UVES data; thus, for them we employed highresolution $(R=100,000)$, high $\mathrm{S} / \mathrm{N}(\sim 400)$ spectra obtained with the HIRES spectrograph at Keck, which covers the optical spectra (388-800 nm) using a mosaic of three CCDs. The asteroid Ceres was used to obtain a solar spectrum for our HIRES observations. In Meléndez et al. (2012) we made a detailed comparison of both UVES and HIRES observations of 18 Sco relative to the Sun and concluded that there is an excellent agreement between both data sets, resulting in negligible abundance differences (mean difference of $0.002 \pm 0.001$ dex and element-toelement scatter $\sigma=0.005 \mathrm{dex}$ ); thus we complement our UVES equivalent-width measurements with HIRES data when needed.

Data reductions of the UVES and HIRES spectra are described in Monroe et al. (2013) and Meléndez et al. (2012), respectively. A comparison of the reduced UVES spectra of $18 \mathrm{Sco}$ and the Sun is shown around 6,085 $($ Figure 1) and 5,320 (Figure 2). As can be seen in Figure 1, overall the spectrum of 18 Sco is very similar to the Sun's, as expected for a solar twin, yet, when a closer look is taken for the lines of neutron-capture elements, a clear enhancement is seen in $18 \mathrm{Sco}$, as shown for example in Figure 2 for the 5,319.8 $\AA \mathrm{Nd}$ II line.

\section{ABUNDANCE ANALYSIS}

The abundance analysis closely follows our recent highprecision studies on the solar twins HIP 56948 (Meléndez et al. 2012) and HIP 102152 (Monroe et al. 2013). We measured the equivalent widths (EW) automatically with the Automatic Routine for line Equivalent widths in stellar Spectra (ARES; Sousa et al. 2007) for lines with EW larger than $10 \mathrm{~m} \AA$. Weaker lines and species with fewer than five lines available were measured by hand using IRAF. In further iterations the outliers resulting from the automatic EW measurements are carefully measured by hand, making sure that exactly the same criteria are used in the measurements of 18 Sco and the Sun, i.e., for each line we choose the same continuum definition, and the same interval was selected to fit a Gaussian profile. The main difference in relation to Meléndez et al. (2012) and Monroe et al. (2013) is that we have significantly expanded our line list to obtain more precise results and also to include many heavy elements. For example, in Meléndez et al. (2012) only 54 iron (42 Fe I, 12 Fe II) and 12 chromium (7 Cr I, 5 Cr II) lines were included, while in the present work 98 iron (86 Fe I, 12 Fe II) and 21 chromium (14 Cr I, $7 \mathrm{Cr}$ II) lines are used. In comparison to Monroe et al. (2013), we have 87 additional lines, many of which were included to study the neutron-capture elements.

Because our abundances were estimated from EW, we selected mostly clean lines. For example, the oxygen abundance was estimated using the clean $\mathrm{O}_{\mathrm{I}}$ triplet at $777 \mathrm{~nm}$ rather than the blended forbidden [O I] line at $630 \mathrm{~nm}$. When necessary we used lines somewhat affected by blending, measuring them by using multiple Gaussians with the deblend option of the task splot in IRAF. The list of lines with the differential equivalentwidth measurements is presented in Table 1, except for nitrogen and lithium, which were analyzed by spectral synthesis of an NH feature at $340 \mathrm{~nm}$ and the Li I feature at $670.7 \mathrm{~nm}$, respectively.

We obtain both stellar parameters and elemental abundances through a differential line-by-line analysis (e.g., Meléndez et al. 2012; Monroe et al. 2013; Ramírez et al. 2011, 2014a), using Kurucz ODFNEW model atmospheres (Castelli \& Kurucz 2004) and the 2002 version of MOOG (Sneden 1973). For the Sun we 


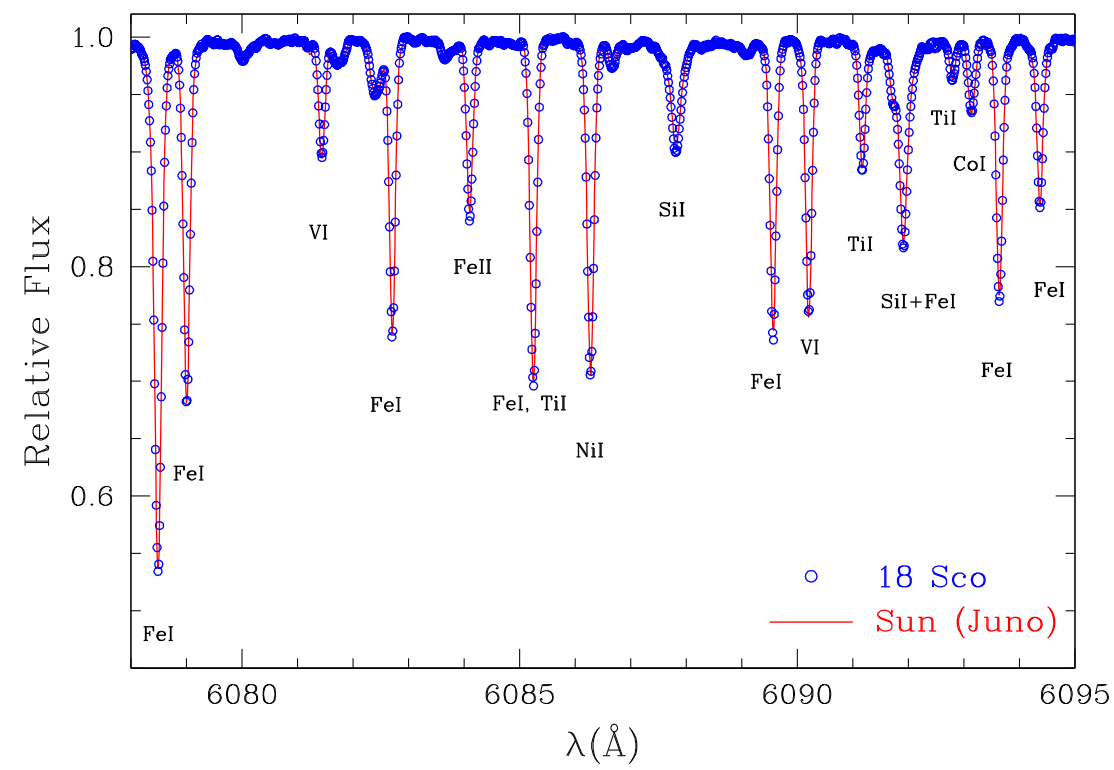

Figure 1. UVES spectra of 18 Sco and the Sun in the 6,078-6,095 A region. Although both stars show similar spectra, their different chemical compositions can be revealed through careful measurements.

(A color version of this figure is available in the online journal.)

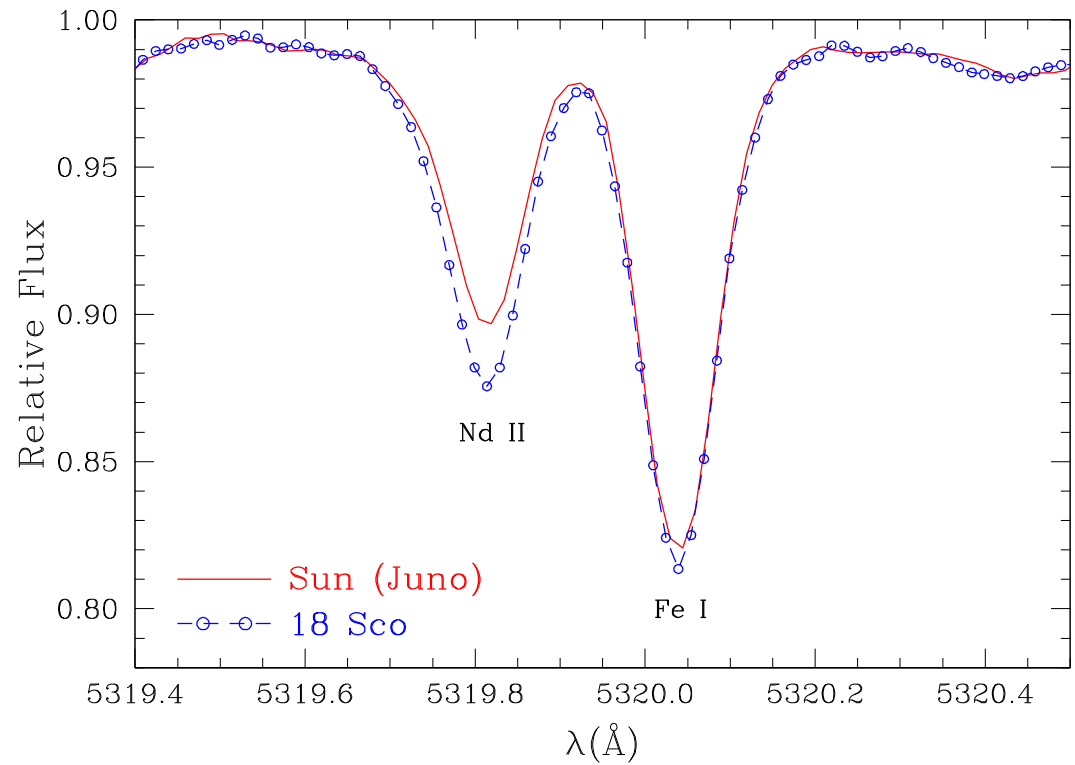

Figure 2. UVES spectra of 18 Sco and the Sun around 5,320 A, showing a clear enhancement in the n-capture element Nd in 18 Sco, relative to the Sun.

(A color version of this figure is available in the online journal.)

Table 1

Adopted Atomic Data and Equivalent Widths

\begin{tabular}{lcccccc}
\hline \hline $\begin{array}{l}\text { Wavelength } \\
(\AA)\end{array}$ & Ion & $\begin{array}{c}\chi_{\text {exc }} \\
(\mathrm{eV})\end{array}$ & $\log g f$ & $C_{6}$ & $\begin{array}{c}\text { EW } \\
18 \text { Sco }\end{array}$ & $\begin{array}{c}\text { EW } \\
\text { Sun }\end{array}$ \\
\hline 5044.211 & 26.0 & 2.8512 & -2.058 & $0.271 \mathrm{E}-30$ & 74.8 & 74.3 \\
5054.642 & 26.0 & 3.640 & -1.921 & $0.468 \mathrm{E}-31$ & 40.9 & 40.5 \\
5127.359 & 26.0 & 0.915 & -3.307 & $0.184 \mathrm{E}-31$ & 97.5 & 96.1 \\
5127.679 & 26.0 & 0.052 & -6.125 & $0.12 \mathrm{E}-31$ & 18.9 & 19.1 \\
5198.711 & 26.0 & 2.223 & -2.135 & $0.461 \mathrm{E}-31$ & 99.3 & 98.1 \\
5225.525 & 26.0 & 0.1101 & -4.789 & $0.123 \mathrm{E}-31$ & 72.5 & 72.1 \\
5242.491 & 26.0 & 3.634 & -0.967 & $0.495 \mathrm{E}-31$ & 88.3 & 86.9 \\
5247.050 & 26.0 & 0.0872 & -4.946 & $0.122 \mathrm{E}-31$ & 67.4 & 66.9 \\
5250.208 & 26.0 & 0.1212 & -4.938 & $0.123 \mathrm{E}-31$ & 66.3 & 65.9 \\
5295.312 & 26.0 & 4.415 & -1.49 & $0.654 \mathrm{E}-30$ & 31.0 & 30.3 \\
\hline
\end{tabular}

(This table is available in its entirety in a machine-readable form in the online journal. A portion is shown here for guidance regarding its form and content.) fixed $T_{\text {eff }}=5,777 \mathrm{~K}$ and $\log g=4.44$ (Cox 2000), and as an initial guess we used a microturbulence velocity of $v_{t}=$ $0.86 \mathrm{~km} \mathrm{~s}^{-1}$ (Monroe et al. 2013). Solar abundances were then computed, and the final solar microturbulence was found by imposing no trend between the abundances of $\mathrm{Fe} I$ lines and reduced equivalent width $\left(\mathrm{EW}_{r}=\mathrm{EW} / \lambda\right)$. We found $v_{t}^{\odot}=$ $0.88 \mathrm{~km} \mathrm{~s}^{-1}$ and used this value and the above $T_{\text {eff }}$ and $\log g$ to compute the reference solar abundances $\left(A_{i}^{\odot}\right)$.

Next, adopting as an initial guess for 18 Sco the solar stellar parameters, we computed abundances for $18 \operatorname{Sco}\left(A_{i}^{*}\right)$ and then differential abundances for each line $i$,

$$
\delta A_{i}=A_{i}^{*}-A_{i}^{\odot} .
$$

The effective temperature is found by imposing the differential excitation equilibrium of $\delta A_{i}$ for Fe I lines:

$$
d\left(\delta A_{i}^{\mathrm{Fe} \mathrm{I}}\right) / d\left(\chi_{\mathrm{exc}}\right)=0,
$$



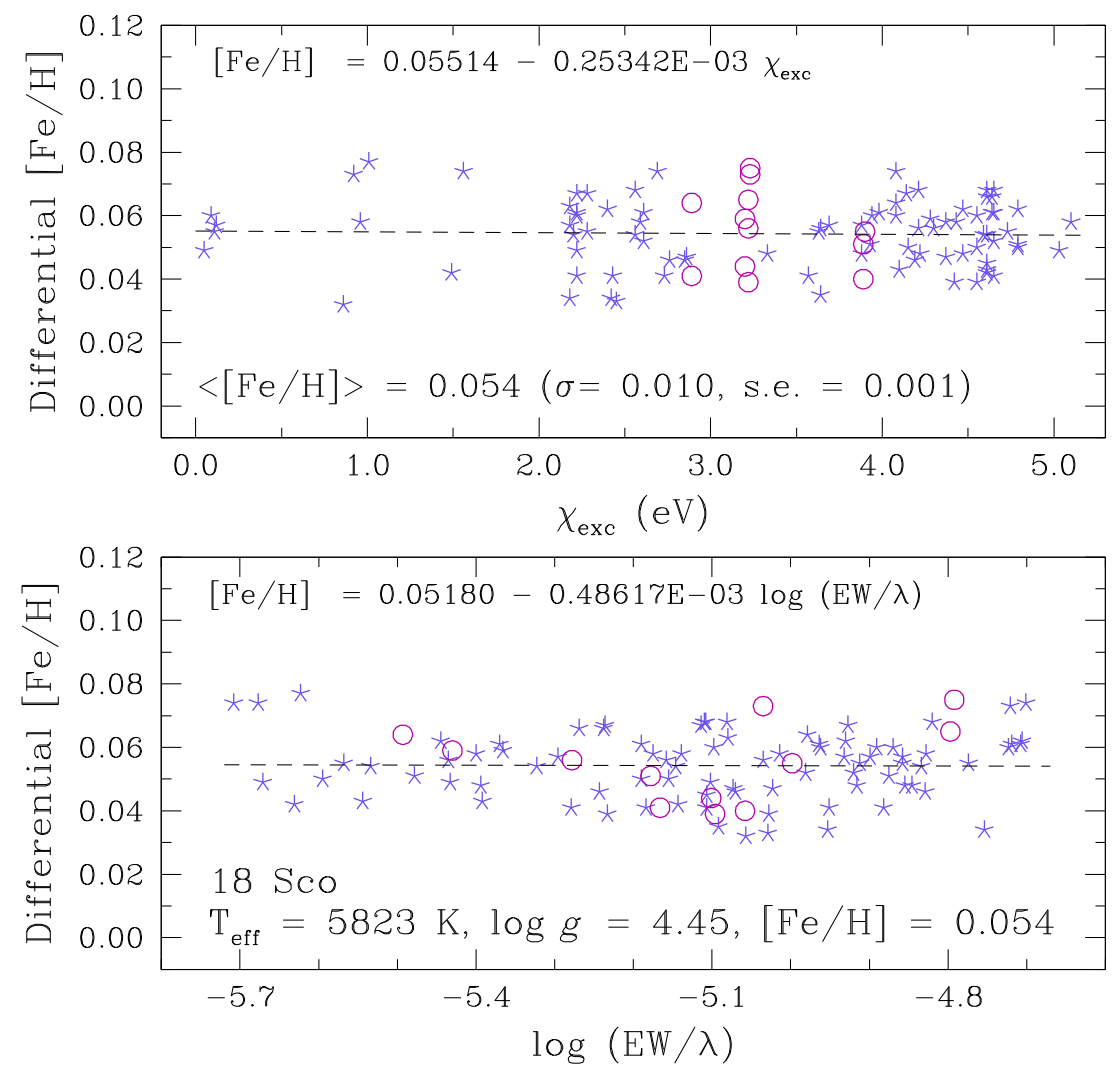

Figure 3. Differential iron abundances vs. excitation potential (top panel) and reduced equivalent width (bottom panel) of Fe I (stars) and Fe II (circles) lines. The dashed lines show fits to the Fe I lines. The solution is achieved when the slopes are equal or smaller than one-third of the error bar in the slopes and when the mean abundance of $\mathrm{Fe}$ II agrees with the mean abundance of Fe I within one-third of the combined error bar.

(A color version of this figure is available in the online journal.)

while the differential ionization equilibrium of $\mathrm{Fe}$ I and Fe II lines was used to determine the surface gravity:

$$
\left\langle\delta A_{i}^{\mathrm{Fe} \mathrm{II}}\right\rangle-\left\langle\delta A_{i}^{\mathrm{Fe} \mathrm{I}}\right\rangle=0 .
$$

The microturbulence velocity, $v_{t}$, was obtained when the differential $\mathrm{Fe}$ I abundances $\delta A_{i}^{\mathrm{Fe}}$ showed no dependence with the logarithm of the reduced equivalent width:

$$
d\left(\delta A_{i}^{\mathrm{Fe}}\right) / d\left(\log _{10}\left(E W_{r}\right)\right)=0 .
$$

The spectroscopic solution is reached when the three conditions above (Equations (2)-(4)) are satisfied simultaneously to better than $\sim$ one-third the error bars in the slopes of Equations (2) and (4), and better than $\sim$ one-third of the error bar of the quadratic sum of the error bars of $\mathrm{Fe}_{\mathrm{I}}$ and $\mathrm{Fe}$ II lines for Equation (3). ${ }^{10}$ We preferred to adopt these convergence criteria based on the observational error bars rather than using fixed values. Also, the metallicity obtained from the iron lines must be the same as that of the input model atmosphere (within 0.01 dex).

We emphasize that our iron line list was built to minimize potential correlations between the atmospheric parameters, by including lines of different line strengths at a given excitation potential and by having, as much as possible, a homogeneous distribution of lines with excitation potential. In addition, we keep in our line list only iron lines that could be reliably measured at high precision at our spectral resolution.

\footnotetext{
10 When it was difficult to achieve convergence using the criteria of one-third of the error bars, we relaxed our criteria to solutions better than one-half of the error bars.
}

The differential spectroscopic equilibrium (Figure 3$)^{11}$ results in the following stellar parameters: $T_{\text {eff }}=5,823 \pm 6 \mathrm{~K} \mathrm{(46 \pm}$ $6 \mathrm{~K}$ hotter than the Sun), $\log g=4.45 \pm 0.02 \mathrm{dex}(+0.01 \pm$ 0.02 dex above the Sun), $[\mathrm{Fe} / \mathrm{H}]=0.054 \pm 0.005 \mathrm{dex}$, and $\Delta v_{t}=+0.02 \pm 0.01 \mathrm{~km} \mathrm{~s}^{-1}$ higher than solar. The above errors include both the measurement uncertainties (from the errors in the slopes and the errors in the iron abundances) and the degeneracies in the stellar parameters, by estimating how the error in a given stellar parameter affects the uncertainty in the others. For example, besides the uncertainty in $\log g$ due to the errors in $\mathrm{Fe}$ I and Fe II, we estimated systematic errors in $\log g$ due to changes in $\mathrm{Fe}$ II-Fe I owing to the uncertainties in $T_{\text {eff }}, v_{t}$ and $[\mathrm{Fe} / \mathrm{H}]$.

In Meléndez et al. (2012) we found that the small differential non-LTE corrections to Fe I lines in the solar twin HIP 56948 do not affect the excitation temperature derived in LTE. Here we also computed NLTE corrections for Fe I as in Bergemann et al. (2012). Again, the differential NLTE corrections are so small that they do not have any impact on our spectroscopic solution. Notice that, within the error bars, the differential ionization equilibrium is satisfied also by $\mathrm{Cr}$, $\mathrm{Ti}$, and $\mathrm{Sc}$, as shown in Figure 4. This good agreement among different species reinforces our results.

Our stellar parameters are in excellent agreement with those independently determined by Monroe et al. (2013), who found

\footnotetext{
11 Notice that the strongest iron lines do not have a significant impact on our final stellar parameters. If we remove the lines with $\log (\mathrm{EW} / \lambda)>-4.8$, the spectroscopic equilibrium would be preserved for $T_{\text {eff }}$ and $\log g$, but only at the $1 \sigma$ level for the microturbulence. The spectroscopic equilibrium would be fully recovered by changing $v_{t}$ by only $-0.01 \mathrm{~km} \mathrm{~s}^{-1}$, without any impact on both $T_{\text {eff }}$ and $\log g$. The resulting $[\mathrm{Fe} / \mathrm{H}]$ would be only 0.002 dex higher.
} 


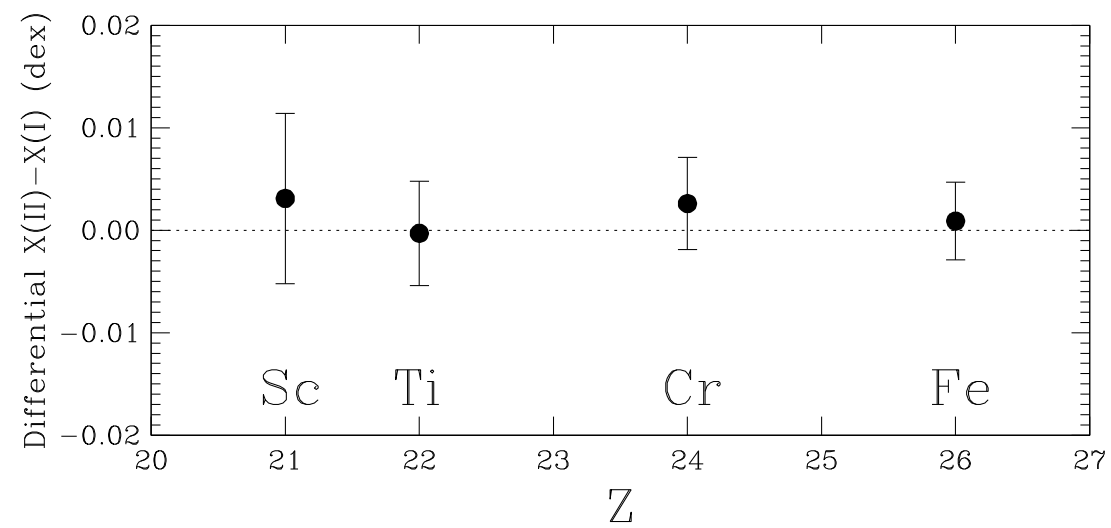

Figure 4. Singly ionized minus neutral differential abundances of $\mathrm{Fe}, \mathrm{Cr}, \mathrm{Ti}$, and Sc. The surface gravity found by the ionization equilibrium of iron also satisfies, within the error bars, the ionization equilibrium of $\mathrm{Sc}, \mathrm{Ti}$, and $\mathrm{Cr}$.

Table 2

Comparison of Stellar Parameters of $18 \mathrm{Sco}$

\begin{tabular}{lclllll}
\hline \hline $\begin{array}{l}T_{\text {eff }} \\
(\mathrm{K})\end{array}$ & $\begin{array}{c}\text { Error } \\
(\mathrm{K})\end{array}$ & $\begin{array}{l}\log g \\
(\mathrm{dex})\end{array}$ & $\begin{array}{l}\text { Error } \\
(\mathrm{dex})\end{array}$ & $\begin{array}{c}{[\mathrm{Fe} / \mathrm{H}]} \\
(\mathrm{dex})\end{array}$ & $\begin{array}{l}\text { Error } \\
(\mathrm{dex})\end{array}$ & \multicolumn{1}{c}{ Source } \\
\hline 5823 & 6 & 4.45 & 0.02 & 0.054 & 0.005 & This work \\
5816 & 4 & 4.45 & 0.01 & 0.053 & 0.003 & Ramírez et al. (2014b) \\
5824 & 5 & 4.45 & 0.02 & 0.055 & 0.010 & Monroe et al. (2013) \\
5810 & 12 & 4.46 & 0.04 & 0.05 & 0.01 & Tsantaki et al. (2013) \\
5831 & 10 & 4.46 & 0.02 & 0.06 & 0.01 & Meléndez et al. (2012) \\
5817 & 30 & 4.45 & 0.13 & 0.05 & 0.05 & da Silva et al. (2012) \\
5826 & 5 & 4.45 & 0.01 & 0.06 & 0.01 & Takeda \& Tajitsu (2009) \\
5840 & 20 & 4.45 & 0.04 & 0.07 & 0.02 & Meléndez et al. (2009) \\
5848 & 46 & 4.46 & 0.06 & 0.06 & 0.02 & Ramírez et al. (2009a) \\
5818 & 13 & 4.45 & 0.02 & 0.04 & 0.01 & Sousa et al. (2008) \\
5834 & 36 & 4.45 & 0.05 & 0.04 & 0.02 & Meléndez \& Ramírez (2007) \\
5822 & 4 & 4.451 & 0.006 & 0.053 & 0.004 & Weighted mean from the literature
\end{tabular}

a $T_{\text {eff }}$ only $1 \mathrm{~K}$ hotter, exactly the same $\log g$ and $v_{t}$, and [Fe/H] only 0.001 dex higher, and by Takeda \& Tajitsu (2009), who determined $T_{\text {eff }}=5,826 \pm 5 \mathrm{~K}, \log g=4.45 \pm 0.01 \mathrm{dex}$, and $[\mathrm{Fe} / \mathrm{H}]=0.06 \pm 0.01$ dex, using high-resolution $(R=90,000)$, high $\mathrm{S} / \mathrm{N}(\sim 1,000$ at $600 \mathrm{~nm})$ High-Definition Spectrograph (HDS)/Subaru spectra. Our results are also in firm agreement with stellar parameters recently determined by Ramírez et al. (2014b) using several high-resolution $(R=65,000-83,000)$, high $\mathrm{S} / \mathrm{N}(=400)$ spectra taken with the MIKE spectrograph at the Magellan telescope, $T_{\text {eff }}=5816 \pm 4 \mathrm{~K}, \log g=4.45 \pm$ $0.01 \mathrm{dex}$, and $[\mathrm{Fe} / \mathrm{H}]=0.053 \pm 0.003$. Also, there is a $\operatorname{good}$ agreement with other results found in the literature, as well as an exceptional accord with their weighted mean value, $T_{\text {eff }}=$ $5822 \pm 4 \mathrm{~K}, \log g=4.45 \pm 0.01 \mathrm{dex}$, and $[\mathrm{Fe} / \mathrm{H}]=0.053 \pm$ 0.004 , as shown in Table 2.

We took hyperfine structure (HFS) into account for 11 elements. The calculation is performed including HFS for each individual line, and then a differential line-by-line analysis is performed. Also, isotopic splitting was taken into account for the heavier elements. For $\mathrm{V}, \mathrm{Mn}, \mathrm{Ag}, \mathrm{Ba}, \mathrm{La}$, and $\mathrm{Pr}$ the combined HFS+isotopic splitting is a minor differential correction $(\leqslant 0.002 \mathrm{dex})$, but for $\mathrm{Co}$ and $\mathrm{Cu}$ the differential correction amounts to 0.004 dex, for $\mathrm{Y}$ the correction is $0.005 \mathrm{dex}$, and for $\mathrm{Yb}$ it is very large at $0.023 \mathrm{dex}$. The most dramatic case is for $\mathrm{Eu}$, for which neglecting the corrections would result in an error of 0.155 dex in the differential abundances.

As shown in Meléndez et al. (2012) and Monroe et al. (2013), differential NLTE effects in solar twins relative to the Sun are minor. Here, we consider differential NLTE corrections for elements showing the largest differential corrections in our previous works, Mn (Bergemann \& Gehren 2008) and Cr (Bergemann \& Cescutti 2010), but the largest differential correction is only $0.003 \mathrm{dex}$ for $\mathrm{Mn}$. As mentioned above, differential NLTE effects on Fe (Bergemann et al. 2012) were also estimated to check for potential systematics in our differential stellar parameters, but there is no impact in our solutions.

Our differential abundances (which are based on EW measured by J. Meléndez) are in excellent agreement with those obtained using an independent set of EW measurements in 18 Sco by Monroe et al. (2013), with an average abundance difference of 0.002 dex (J.M.'s measurements in this paper; Monroe et al. 2013) and an element-to-element scatter of only 0.005 dex. Another independent set of EW measurements obtained by M. Tucci Maia (which were obtained fully by hand, unlike the measurements done by J.M and T.M., which used ARES first and then remeasured the outliers by hand) results in abundances with a difference from our work of 0.002 dex and scatter of only 0.004 dex. These comparisons and our previous testing in Meléndez et al. (2012), for which we obtained an element-toelement scatter of $\sigma=0.005$ dex, in the similarity of HIRES and UVES abundances of 18 Sco minus the Sun, suggest that careful differential measurements can achieve a precision of about 0.005 dex in differential abundances.

The measurement errors are adopted as the standard error of the differential abundances, except for elements with just a single line, in which case we adopted as observational error the standard deviation of five differential EW measurements performed with somewhat different criteria. The typical 
Table 3

Differential Abundances of 18 Sco Relative to the Sun and their Errors

\begin{tabular}{|c|c|c|c|c|c|c|c|c|}
\hline Element & $\begin{array}{l}{[\mathrm{X} / \mathrm{H}]} \\
(\mathrm{dex})\end{array}$ & $\begin{array}{l}\Delta T_{\text {eff }} \\
+6 \mathrm{~K} \\
(\mathrm{dex})\end{array}$ & $\begin{array}{c}\Delta \log g \\
+0.02 \text { dex } \\
\quad(\operatorname{dex})\end{array}$ & $\begin{array}{c}\Delta v_{t} \\
+0.01 \mathrm{~km} \mathrm{~s}^{-1} \\
(\mathrm{dex})\end{array}$ & $\begin{array}{c}\Delta[\mathrm{M} / \mathrm{H}] \\
+0.01 \mathrm{dex} \\
\quad(\mathrm{dex})\end{array}$ & $\begin{array}{l}\text { Param }^{a} \\
(\text { dex })\end{array}$ & $\begin{array}{l}\mathrm{Obs}^{\mathrm{b}} \\
(\mathrm{dex})\end{array}$ & $\begin{array}{l}\text { Total }^{\mathrm{c}} \\
(\mathrm{dex})\end{array}$ \\
\hline $\mathrm{C}$ & -0.009 & -0.004 & 0.004 & 0.000 & 0.000 & 0.006 & 0.007 & 0.009 \\
\hline $\mathrm{N}$ & 0.003 & 0.002 & 0.004 & 0.006 & 0.007 & 0.010 & 0.013 & 0.017 \\
\hline $\mathrm{O}$ & -0.003 & -0.005 & 0.000 & -0.001 & 0.002 & 0.005 & 0.006 & 0.008 \\
\hline $\mathrm{Na}$ & 0.025 & 0.003 & 0.000 & -0.001 & 0.000 & 0.003 & 0.003 & 0.004 \\
\hline $\mathrm{Mg}$ & 0.039 & 0.004 & -0.001 & -0.002 & 0.000 & 0.005 & 0.008 & 0.009 \\
\hline $\mathrm{Al}$ & 0.034 & 0.002 & -0.002 & -0.001 & 0.000 & 0.003 & 0.006 & 0.007 \\
\hline $\mathrm{Si}$ & 0.047 & 0.001 & 0.001 & -0.001 & 0.001 & 0.002 & 0.002 & 0.003 \\
\hline $\mathrm{S}$ & 0.016 & -0.003 & 0.003 & 0.000 & 0.001 & 0.004 & 0.007 & 0.008 \\
\hline $\mathrm{K}$ & 0.041 & 0.005 & -0.007 & -0.002 & 0.001 & 0.009 & 0.005 & 0.010 \\
\hline $\mathrm{Ca}$ & 0.057 & 0.004 & -0.003 & -0.002 & 0.000 & 0.005 & 0.003 & 0.006 \\
\hline $\mathrm{Sc}^{\mathrm{d}}$ & 0.047 & 0.000 & 0.007 & -0.002 & 0.003 & 0.008 & 0.004 & 0.009 \\
\hline $\mathrm{Ti}^{\mathrm{d}}$ & 0.051 & 0.006 & 0.001 & -0.001 & -0.001 & 0.006 & 0.002 & 0.007 \\
\hline V & 0.041 & 0.006 & 0.002 & -0.001 & -0.001 & 0.006 & 0.003 & 0.007 \\
\hline $\mathrm{Cr}^{\mathrm{d}}$ & $0.056^{\mathrm{e}}$ & 0.005 & -0.001 & -0.002 & 0.000 & 0.005 & 0.001 & 0.006 \\
\hline Mn & $0.041^{\mathrm{e}}$ & 0.005 & -0.002 & -0.002 & 0.001 & 0.006 & 0.001 & 0.006 \\
\hline $\mathrm{Fe}^{\mathrm{d}}$ & 0.054 & 0.004 & -0.001 & -0.002 & 0.000 & 0.005 & 0.001 & 0.005 \\
\hline Co & 0.027 & 0.004 & 0.002 & -0.001 & 0.000 & 0.005 & 0.003 & 0.005 \\
\hline $\mathrm{Ni}$ & 0.041 & 0.003 & 0.000 & -0.002 & 0.001 & 0.004 & 0.002 & 0.004 \\
\hline $\mathrm{Cu}$ & 0.032 & 0.003 & 0.001 & -0.002 & 0.001 & 0.004 & 0.004 & 0.006 \\
\hline $\mathrm{Zn}$ & 0.017 & 0.001 & 0.002 & -0.002 & 0.003 & 0.004 & 0.002 & 0.005 \\
\hline $\mathrm{Sr}$ & 0.097 & 0.006 & 0.000 & -0.004 & -0.001 & 0.007 & 0.011 & 0.013 \\
\hline $\mathrm{Y}$ & 0.099 & 0.001 & 0.006 & -0.005 & 0.004 & 0.009 & 0.007 & 0.011 \\
\hline $\mathrm{Zr}$ & 0.102 & 0.001 & 0.008 & -0.003 & 0.003 & 0.009 & 0.010 & 0.014 \\
\hline Mo & 0.115 & 0.001 & 0.002 & -0.001 & 0.000 & 0.002 & 0.015 & 0.015 \\
\hline $\mathrm{Ru}$ & 0.143 & 0.001 & 0.002 & -0.002 & 0.000 & 0.003 & 0.028 & 0.028 \\
\hline $\mathrm{Pd}$ & 0.125 & 0.001 & 0.003 & -0.002 & 0.001 & 0.004 & 0.010 & 0.011 \\
\hline $\mathrm{Ag}$ & 0.126 & 0.001 & 0.002 & -0.004 & 0.001 & 0.005 & 0.001 & 0.005 \\
\hline $\mathrm{Ba}$ & 0.118 & 0.002 & 0.002 & -0.004 & 0.005 & 0.007 & 0.006 & 0.009 \\
\hline $\mathrm{La}$ & 0.135 & 0.001 & 0.009 & 0.000 & 0.003 & 0.010 & 0.006 & 0.011 \\
\hline $\mathrm{Ce}$ & 0.117 & 0.001 & 0.009 & -0.001 & 0.003 & 0.010 & 0.006 & 0.011 \\
\hline Pr & 0.134 & 0.001 & 0.009 & 0.000 & 0.003 & 0.010 & 0.014 & 0.017 \\
\hline $\mathrm{Nd}$ & 0.153 & 0.002 & 0.009 & -0.001 & 0.003 & 0.010 & 0.009 & 0.013 \\
\hline $\mathrm{Sm}$ & 0.165 & 0.002 & 0.009 & -0.001 & 0.003 & 0.010 & 0.018 & 0.020 \\
\hline $\mathrm{Eu}$ & 0.187 & 0.002 & 0.007 & -0.004 & 0.004 & 0.009 & 0.030 & 0.031 \\
\hline Gd & 0.171 & 0.002 & 0.010 & -0.001 & 0.004 & 0.011 & 0.003 & 0.011 \\
\hline Dy & 0.178 & 0.002 & 0.009 & -0.002 & 0.004 & 0.010 & 0.012 & 0.016 \\
\hline $\mathrm{Yb}$ & 0.156 & 0.003 & 0.003 & -0.005 & 0.005 & 0.008 & 0.012 & 0.015 \\
\hline
\end{tabular}

Notes.

${ }^{\text {a }}$ Errors due to stellar parameters.

b Observational errors.

c Quadric sum of errors due to observational and stellar parameter uncertainties.

${ }^{\mathrm{d}}$ For Sc, Ti, Cr, and Fe, the systematic errors due to stellar parameters refer to Sc II, Ti I, Cr I, and Fe I, respectively.

${ }^{e}$ NLTE abundances are reported for these elements. LTE abundances are $[\mathrm{Cr} / \mathrm{H}]=0.058,[\mathrm{Mn} / \mathrm{H}]=0.044$.

measurement uncertainties in the differential abundances of the lighter elements $(Z \leqslant 30)$ are about 0.004 dex, in good agreement with the measurement errors discussed above. Including the systematic errors due to uncertainties in the stellar parameters, the total error is about 0.007 dex. The differential abundances for each element and their errors are given in Table 3.

\section{MASS, AGE, ROTATION, AND LITHIUM}

We determine the mass and age of 18 Sco using our precise stellar parameters $\left(T_{\text {eff }}=5823 \pm 6 \mathrm{~K}, \log g=4.45 \pm 0.02 \mathrm{dex}\right.$, $[\mathrm{Fe} / \mathrm{H}]=0.054 \pm 0.005 \mathrm{dex}$ ) and Yonsei-Yale isochrones (Yi et al. 2001; Kim et al. 2002; Demarque et al. 2004). The method, which uses the stellar parameters, their error bars, and probability distribution functions, is described in detail in Meléndez et al. (2012) and Chanamé \& Ramírez (2012).
The method was calibrated to reproduce the solar values, as described in Meléndez et al. (2012).

We obtain an age of $2.9_{-1.0}^{+1.1} \mathrm{Gyr}$ for $18 \mathrm{Sco}$, i.e., $1.6 \mathrm{Gyr}$ younger than the Sun, for which we derived an age of $4.5 \mathrm{Gyr}$ using the same set of isochrones (Meléndez et al. 2012). Stellar ages can be well-constrained from isochrones, provided that stellar parameters are known with extreme precision, as shown in Figure 5(a), where we compare our stellar parameters and error bars to the Yonsei-Yale isochrones. We show in Figure 5(b) that Padova isochrones ${ }^{12}$ (Bressan et al. 2012) are compatible with the relative ages between the Sun and 18 Sco obtained from Yonsei-Yale isochrones. Our age agrees well with the value found by do Nascimento et al. (2009), 2.89+-1.09 Gyr, using lithium abundances and stellar parameters. Within the error

\footnotetext{
12 http://stev.oapd.inaf.it/cgi-bin/cmd
} 


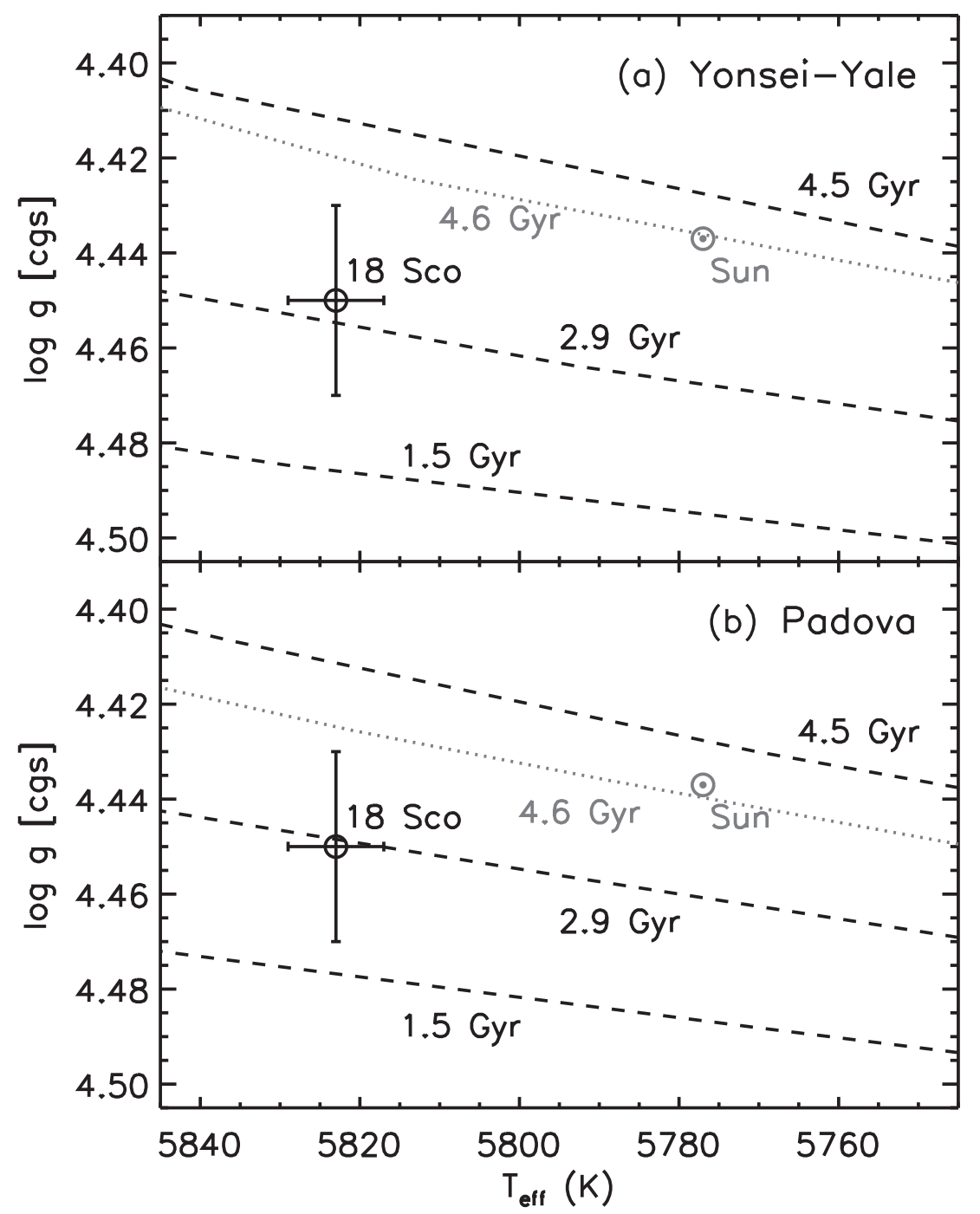

Figure 5. Location of the Sun and 18 Sco on the HR diagram. (a) Yonsei-Yale isochrones of $[\mathrm{Fe} / \mathrm{H}]=+0.05$ (the metallicity of $18 \mathrm{Sco}$ ) and age $=1.5$, 2.9 , and $4.5 \mathrm{Gyr}$ are shown (dashed lines) along with a solar metallicity isochrone of solar age (dotted line). The high precision of our derived stellar parameters for 18 Sco allows us to infer a reasonable estimate of its age from the theoretical isochrones, even though they are densely packed in this main-sequence region. (b) As above for Padova isochrones, showing consistent results for the relative ages between $18 \mathrm{Sco}$ and the Sun.

bars, our age also agrees with that determined by Li et al. (2012), $3.66_{-0.50}^{+0.44} \mathrm{Gyr}$, using different constraints (stellar parameters, lithium abundance, rotation, and average large-frequency separation). Notice, however, that the adopted stellar parameters by Li et al. (2012) are not as precise as those reported in this work. We are currently modeling our High Accuracy Radial velocity Planet Searcher (HARPS) seismic observations of 18 Sco to obtain even better constraints on its age.

Following Meléndez et al. (2012), we obtain $v \sin i_{18 \mathrm{Sco}} / v$ $\sin i_{\odot}=1.069 \pm 0.029$. Adopting $v \sin i_{\odot}=1.90 \mathrm{~km} \mathrm{~s}^{-1}$ for the Sun (Bruning 1984; Saar \& Osten 1997), this implies $v$ sin $i_{18 \mathrm{Sco}}=2.03 \pm 0.05 \mathrm{~km} \mathrm{~s}^{-1}$. The higher rotation rate in $18 \mathrm{Sco}$ is compatible with its younger age. Fortunately, the rotation period has been determined for this star, $P=22.7 \pm 0.5$ days (Petit et al. 2008), resulting in a rotational age of $3.36 \pm 0.52 \mathrm{Gyr}$ using the rotation-age relationship given in Barnes (2007). This value is in good agreement with our derived isochronal age $\left(2.9_{-1.0}^{+1.1} \mathrm{Gyr}\right)$.

The mass derived here is $1.04 \pm 0.02 M_{\odot}$, which agrees well with the detailed study of $\mathrm{Li}$ et al. (2012), who reported $1.045 M_{\odot}$ using isochrones, $1.030 M_{\odot}$ adding also lithium, and 1.030 , including stellar parameters, lithium, and the mean large- frequency separation. The mass derived by do Nascimento et al. (2009), $1.01 \pm 0.01 M_{\odot}$, is also in agreement with our results within the error bars and with the mass derived using asteroseismology, $1.02 \pm 0.03 M_{\odot}$ (Bazot et al. 2011) and $1.01 \pm$ $0.03 M_{\odot}$ (Bazot et al. 2012). We are performing a more detailed seismic analysis of $18 \mathrm{Sco}$, including also new HARPS observations (M. Bazot et al., in preparation).

The $\mathrm{Li}$ abundance $\left(A_{\mathrm{Li}}^{\mathrm{NLTE}}=1.62 \pm 0.02 \mathrm{dex}\right)$ was derived using the line list presented in Meléndez et al. (2012) and NLTE corrections by Lind et al. (2009), and it is identical to that obtained by Monroe et al. (2013), as the stellar parameters are essentially the same, except for a $1 \mathrm{~K}$ difference in the effective temperature. We refer the reader to Monroe et al. (2013) for further details, but we stress here that our Li abundance fits well the trend of Li depletion with age of several nonstandard solar models (e.g., Charbonnel \& Talon 2005; do Nascimento et al. 2009; Xiong \& Deng 2009; Denissenkov 2010).

\section{COMPANIONS AROUND 18 Sco}

The 18 Sco is included in our HARPS Large Program to search for planets around solar twins; hence we can evaluate whether planets or a binary component are present. 


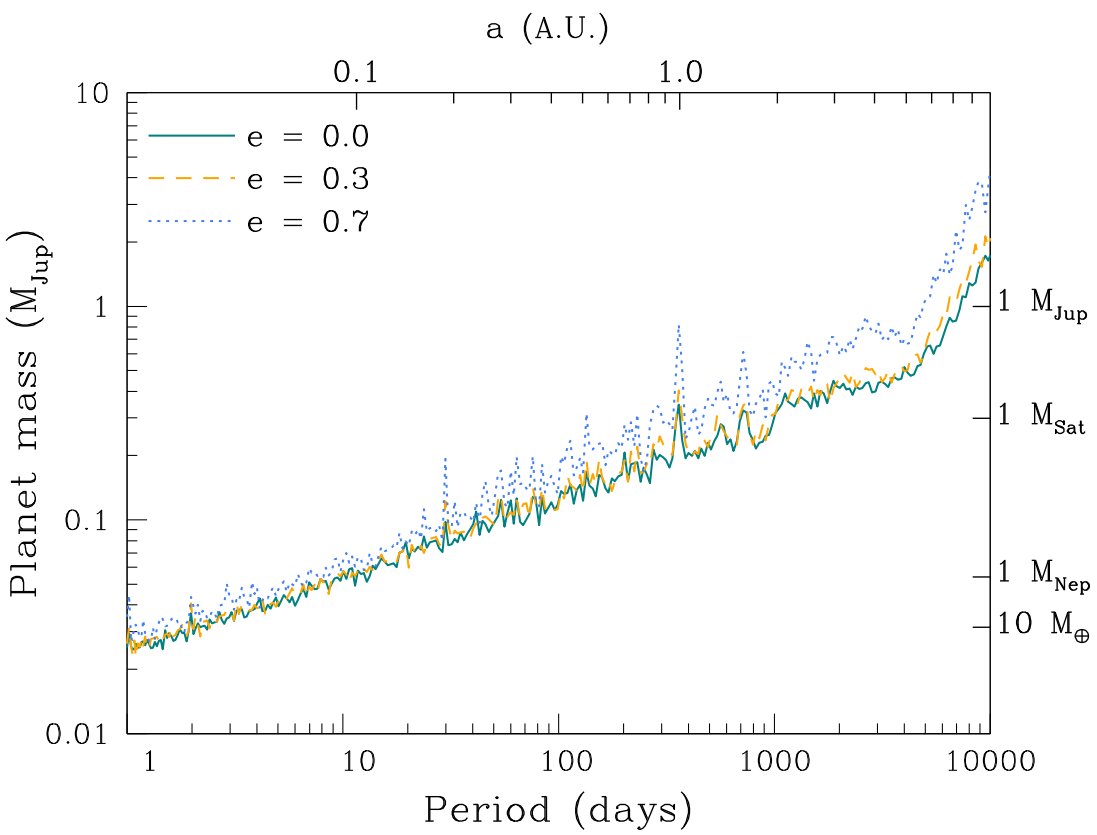

Figure 6. Detection limits based on our HARPS data are shown for different eccentricities (e) as a function of orbital period. Planets above the curves are ruled out.

(A color version of this figure is available in the online journal.)

Radial velocities were obtained with the HARPS instrument and binned to yield one radial velocity (RV) value per night for a total of 59 nights spanning 2004 May to 2014 February. The observations include 20 nights of high-cadence asteroseismic observations without simultaneous reference in addition to 39 nights of observations with simultaneous ThAr reference for instrumental drift correction. The asteroseismic data have a scatter of $1 \mathrm{~m} \mathrm{~s}^{-1}$ throughout the course of a single night. When a moving average is applied to smooth out random noise, a coherent and repeated nightly pattern with an amplitude on the order of $2 \mathrm{~m} \mathrm{~s}^{-1}$ emerges. We conclude that this coherent noise may be instrumental in origin, and we minimize its effect on the data by using only a single data point from each night, obtained by a weighted average of points from the four hours with lowest air mass. In addition, the radial velocities show drifts throughout the course of the two asteroseismic observing runs, which may be instrumental or, in the case of the 2012 May observations, may be a signal from star spots. We make no attempt to remove these drifts due to the uncertainty of their origin.

Activity indices were also calculated for each HARPS spectrum from the $\mathrm{Ca}$ II $\mathrm{H} \& \mathrm{~K}$ lines. The activity cycle of $18 \mathrm{Sco}$ is present in the data, with radial velocities increasing at times of high activity as photospheric convection is suppressed. This variation occurs on a timescale of $7.6 \mathrm{yr}$ in the data, consistent with the previously measured period of seven years from photometry and chromospheric activity (Hall et al. 2007). We remove the effect of the activity cycle on the RVs by fitting and subtracting a linear correlation between radial velocity and continuum-normalized Ca II H \& K flux $\left(S_{H K}\right)$.

The resulting radial velocity measurements were searched for planet signals with no significant detections. We quantify our upper limits on potential planets as follows. We make a flat-line fit to the data and subtract the offset. We then fold all jitter in the radial velocities into the uncertainties on the residuals by scaling them with the reduced chi-squared of the fit. The residuals are resampled randomly with replacement, and a Keplerian signal of fixed planet period and mass is added. If the resulting simulated RVs are inconsistent with a flat-line fit by three or more sigma for $99 \%$ of randomized trials at a given planet period and mass, we consider the planet to be excluded by the data. The resulting exclusion limits rule out sub-Neptune-mass objects out to a period of seven days and Jupiter-mass objects out to approximately $13 \mathrm{yr}$ (Figure 6). As with all ground-based radial velocity observations, these data show aliases on timescales around one day (due to observing nightly) and at approximately 30 and 365 days due to the effects of the lunar cycle and seasonal observability on sampling.

Our HARPS RV measurements also exclude the presence of a binary component, which is consistent with no detection of companions around 18 Sco by high-contrast adaptive optics (AO) imaging (Tanner et al. 2010).

\section{THE COMPLEX ABUNDANCE PATTERN OF 18 Sco}

As can be seen in Figure 7, 18 Sco presents a complex abundance pattern. On top of the typical trend with condensation temperature seen in other solar twins (Meléndez et al. 2009; Ramírez et al. 2009a, 2010), corresponding in Figure 7 to the group of elements with enhancements $[\mathrm{X} / \mathrm{H}] \leqslant 0.06 \mathrm{dex}$ (filled circles), there is a group of elements with much larger enhancements $(0.09<[\mathrm{X} / \mathrm{H}]<0.19 \mathrm{dex}$; filled triangles $)$. All elements in the latter group are neutron-capture elements.

In order to understand the large enhancement of the n-capture elements in $18 \mathrm{Sco}$, we first need to subtract the trend with condensation temperature, as it is probably related to the deficiency of refractory elements in the Sun (Meléndez et al. 2009); besides, the yields of asymptotic giant branch (AGB) stars and supernova $(\mathrm{SN})$ do not produce such a trend (Meléndez et al. 2012). We fit $[\mathrm{X} / \mathrm{H}]$ versus condensation temperature (Lodders 2003) for the lighter elements with $Z \leqslant 30$ because they only seem affected by the condensation temperature. The fit (Figure 8) results in

$$
[\mathrm{X} / \mathrm{H}](Z \leqslant 30)=-0.005+3.485 \times 10^{-5} T_{\text {cond }},
$$

with an element-to-element scatter of only $0.008 \mathrm{dex}$, which is similar to the mean total error $(0.007 \mathrm{dex})$ of our differential 


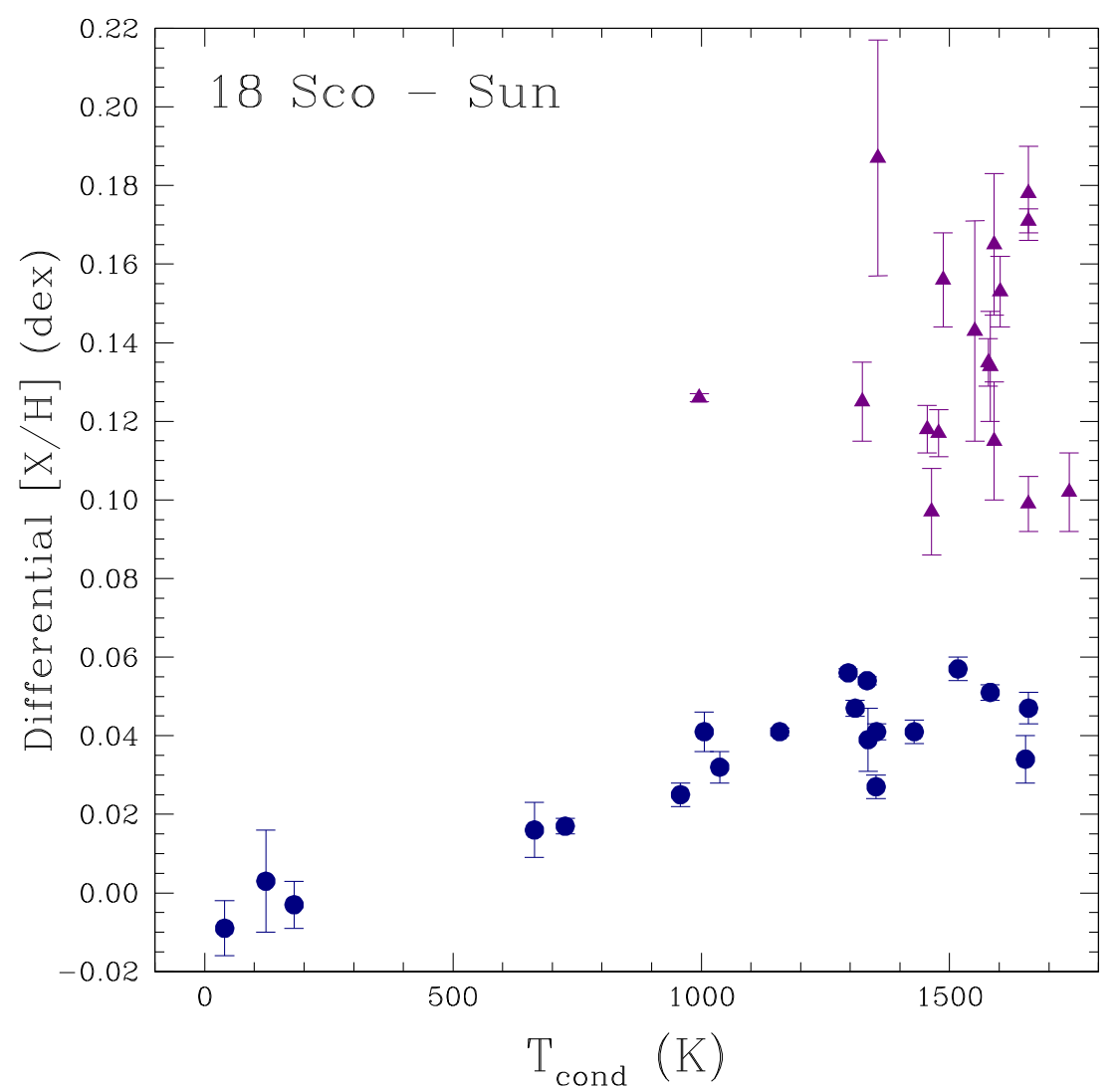

Figure 7. Differential abundances of 18 Sco relative to the Sun. Elements with $[X / H] \leqslant 0.06$ (filled circles) have $Z \leqslant 30$, i.e., zinc and lighter elements, while the elements with $[\mathrm{X} / \mathrm{H}]>0.09$ (filled triangles) have $Z>30$.

(A color version of this figure is available in the online journal.)

abundances for elements with $Z \leqslant 30$ (Table 3), showing thus that our small error bars are realistic. The significance of the slope is $9 \sigma$.

Next, we subtract the above trend from the $[\mathrm{X} / \mathrm{H}]$ abundances:

$$
[\mathrm{X} / \mathrm{H}]_{T}=[\mathrm{X} / \mathrm{H}]-\left(-0.005+3.485 \times 10^{-5} T_{\text {cond }}\right) .
$$

The $[\mathrm{X} / \mathrm{H}]_{T}$ ratios are given in Table 4 and are shown by filled circles in Figure 9.

We first verify if the observed enhancement in the $n$-capture elements is due to pollution by AGB stars. We used a model of a $3 M_{\odot}$ AGB star of $Z=0.01$ (Karakas 2010) ) $^{13}$ and diluted the yields of a small fraction of AGB ejecta $(\leqslant 1 \%$ of material injected) into a $1 M_{\odot}$ protocloud of solar composition (Asplund et al. 2009). Then, we computed the enhancement in the abundance ratios relative to the initial composition of the protocloud, $[\mathrm{X} / \mathrm{H}]_{\mathrm{AGB}}$. A dilution of $0.23 \%$ mass of AGB material matched the observed enhancement in the light $s$-process element ${ }^{14}$ yttrium. The $[\mathrm{X} / \mathrm{H}]_{\mathrm{AGB}}$ ratios are given in Table 4 and are shown by open triangles in Figure 9. As can be seen, a good match cannot be achieved for all of the $\mathrm{n}$-capture elements, showing that there is an additional source for the abundance enhancement. Nevertheless, other $s$-process elements besides $\mathrm{Y}$, such as $\mathrm{Sr}, \mathrm{Zr}$, and $\mathrm{Ba}$, are well fitted; thus,

\footnotetext{
13 Similar models are considered to derive the $s$-process contribution in the solar system (e.g., Arlandini et al. 1999).

14 As common in the literature, we use the terms $s$-process and $r$-process elements, but rigorously speaking that is incorrect because the $s$ and $r$ neutron-capture processes are responsible for the synthesis of isotopes. The $s$-process and $r$-process elements are deduced from decomposition of solar system material.
}

Table 4

Neutron-capture Enhancement in 18 Sco

\begin{tabular}{llllll}
\hline \hline$Z$ & Element & $\begin{array}{c}{[\mathrm{X} / \mathrm{H}]_{T}} \\
(\mathrm{dex})\end{array}$ & $\begin{array}{c}{[\mathrm{X} / \mathrm{H}]_{\mathrm{AGB}}} \\
(\mathrm{dex})\end{array}$ & $s_{\text {Sim }}{ }^{\mathrm{a}}$ & $s_{\mathrm{Bis}} \mathrm{b}$ \\
\hline 38 & $\mathrm{Sr}$ & 0.051 & 0.043 & 0.890 & 0.67 \\
39 & $\mathrm{Y}$ & 0.046 & 0.046 & 0.719 & 0.70 \\
40 & $\mathrm{Zr}$ & 0.046 & 0.056 & 0.809 & 0.64 \\
42 & $\mathrm{Mo}$ & 0.064 & 0.030 & 0.677 & 0.577 \\
44 & $\mathrm{Ru}$ & 0.094 & 0.023 & 0.39 & 0.373 \\
46 & $\mathrm{Pd}$ & 0.084 & 0.032 & 0.445 & 0.531 \\
47 & $\mathrm{Ag}$ & 0.096 & 0.013 & 0.212 & 0.221 \\
56 & $\mathrm{Ba}$ & 0.072 & 0.064 & 0.853 & 0.83 \\
57 & $\mathrm{La}$ & 0.085 & 0.055 & 0.754 & 0.711 \\
58 & $\mathrm{Ce}$ & 0.070 & 0.054 & 0.814 & 0.81 \\
59 & $\mathrm{Pr}$ & 0.084 & 0.032 & 0.492 & 0.49 \\
60 & $\mathrm{Nd}$ & 0.102 & 0.037 & 0.579 & 0.56 \\
62 & $\mathrm{Sm}$ & 0.115 & 0.018 & 0.331 & 0.31 \\
63 & $\mathrm{Eu}$ & 0.145 & 0.003 & 0.027 & 0.06 \\
64 & $\mathrm{Gd}$ & 0.118 & 0.008 & 0.181 & 0.135 \\
66 & $\mathrm{Dy}$ & 0.125 & 0.010 & 0.121 & 0.148 \\
70 & $\mathrm{Yb}$ & 0.109 & 0.024 & 0.318 & 0.399 \\
\hline
\end{tabular}

Notes.

a $s$-process solar system fractions by Simmerer et al. (2004).

b $s$-process solar system fractions by Bisterzo et al. $(2011,2013)$.

the observed enhancement could be due at least partly to AGB stars. In order to find out if the residual enhancement is due to the $r$ process, we estimate its enrichment in 18 Sco by subtracting the AGB contribution

$$
[\mathrm{X} / \mathrm{H}]_{r}=[\mathrm{X} / \mathrm{H}]_{T}-[\mathrm{X} / \mathrm{H}]_{\mathrm{AGB}},
$$




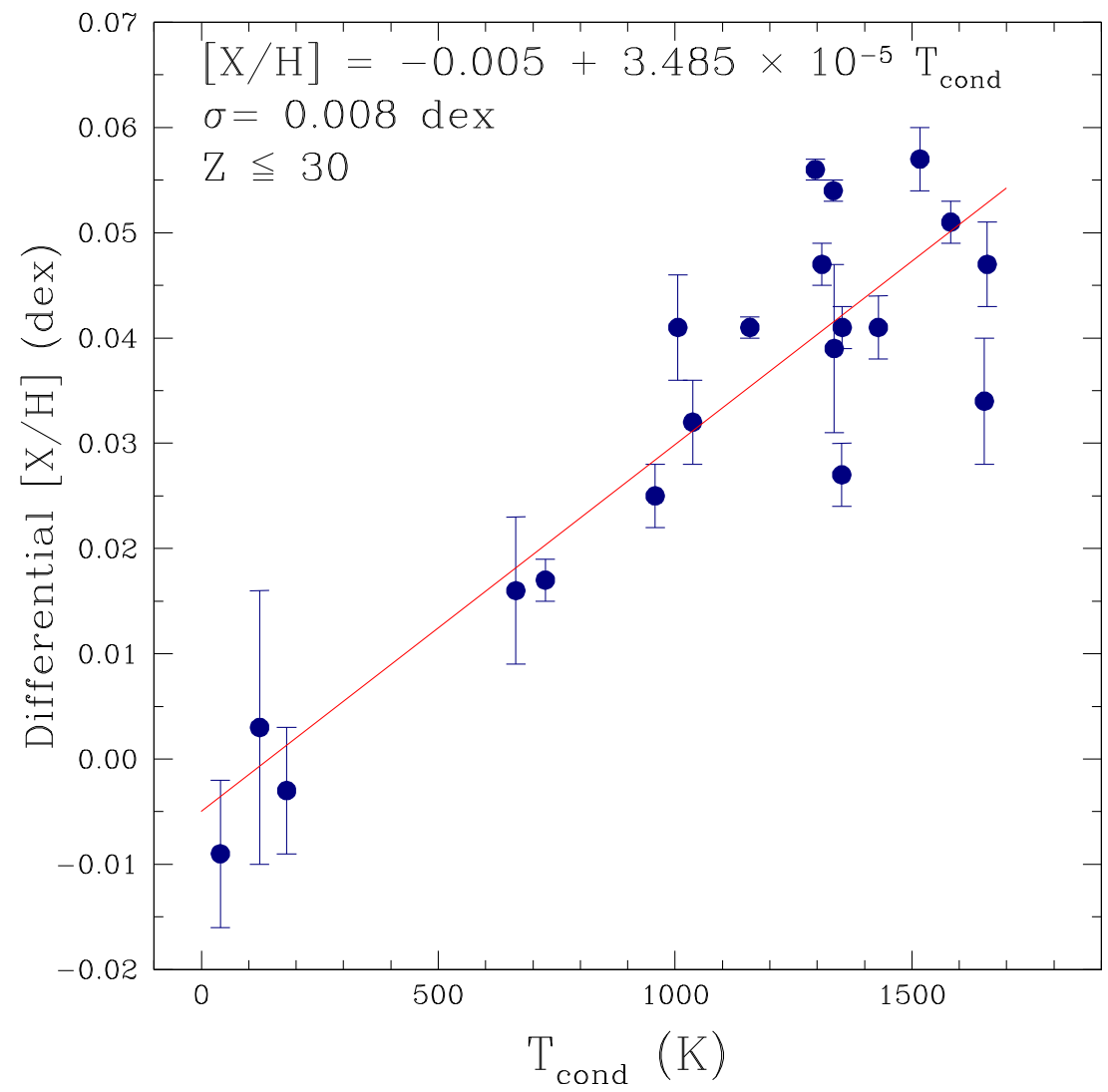

Figure 8. Fit of the trend with condensation temperature for elements with $Z \leqslant 30$. The slope has a significance of $9 \sigma$, and the element-to-element scatter from the fit is only 0.008 dex.

(A color version of this figure is available in the online journal.)

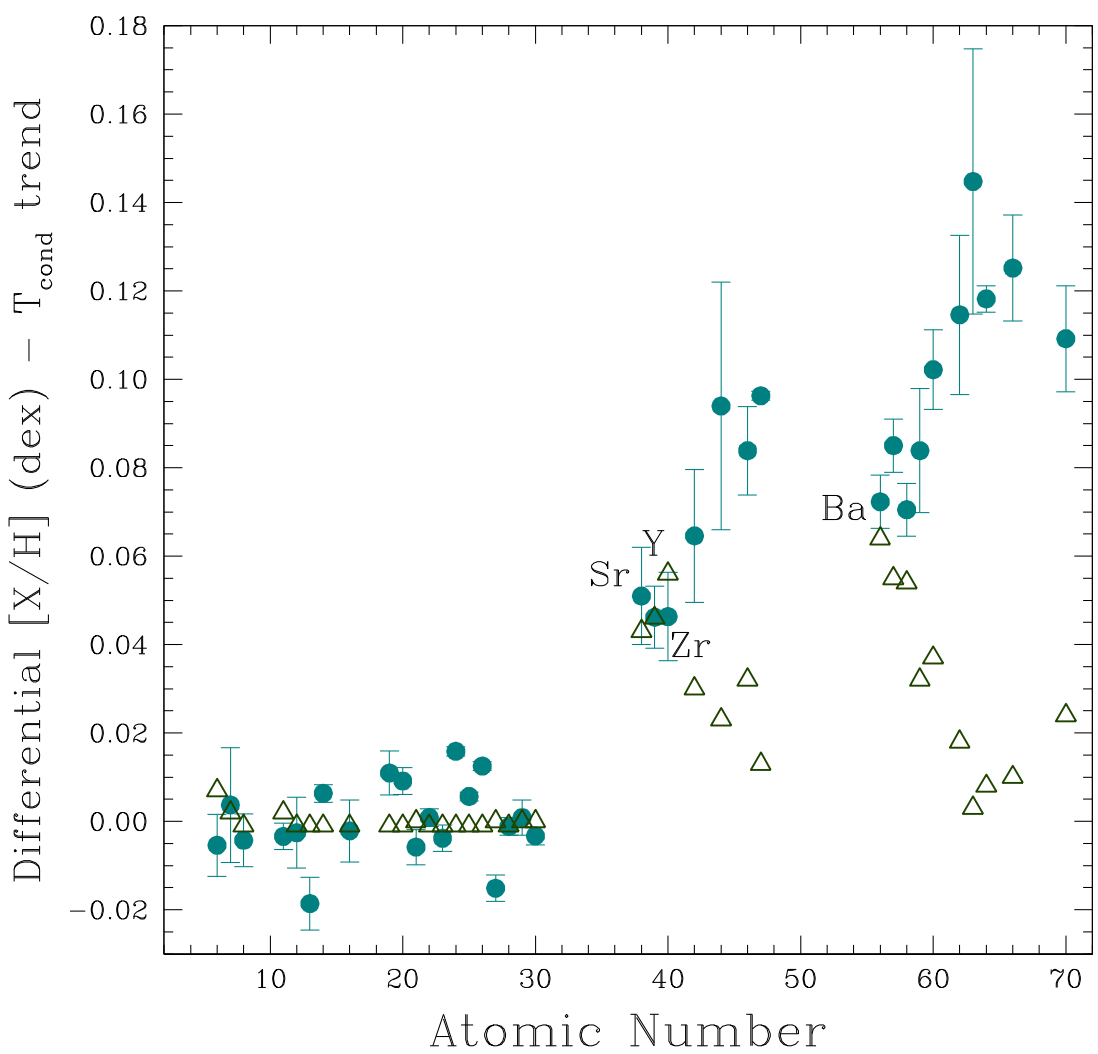

Figure 9. Filled circles are the $[\mathrm{X} / \mathrm{H}]$ ratios in $18 \mathrm{Sco}$ after they have been subtracted from the condensation temperature trend shown in Figure 8 . Elements with $Z \leqslant 30$ now have abundance ratios close to zero, but the neutron-capture elements remain enhanced. The open triangles represent the effect of pollution by an AGB star (see the text for details). Although a good match can be seen for the s-process elements $\mathrm{Sr}, \mathrm{Y}, \mathrm{Zr}$, and $\mathrm{Ba}$, there is a disagreement for other elements.

(A color version of this figure is available in the online journal.) 


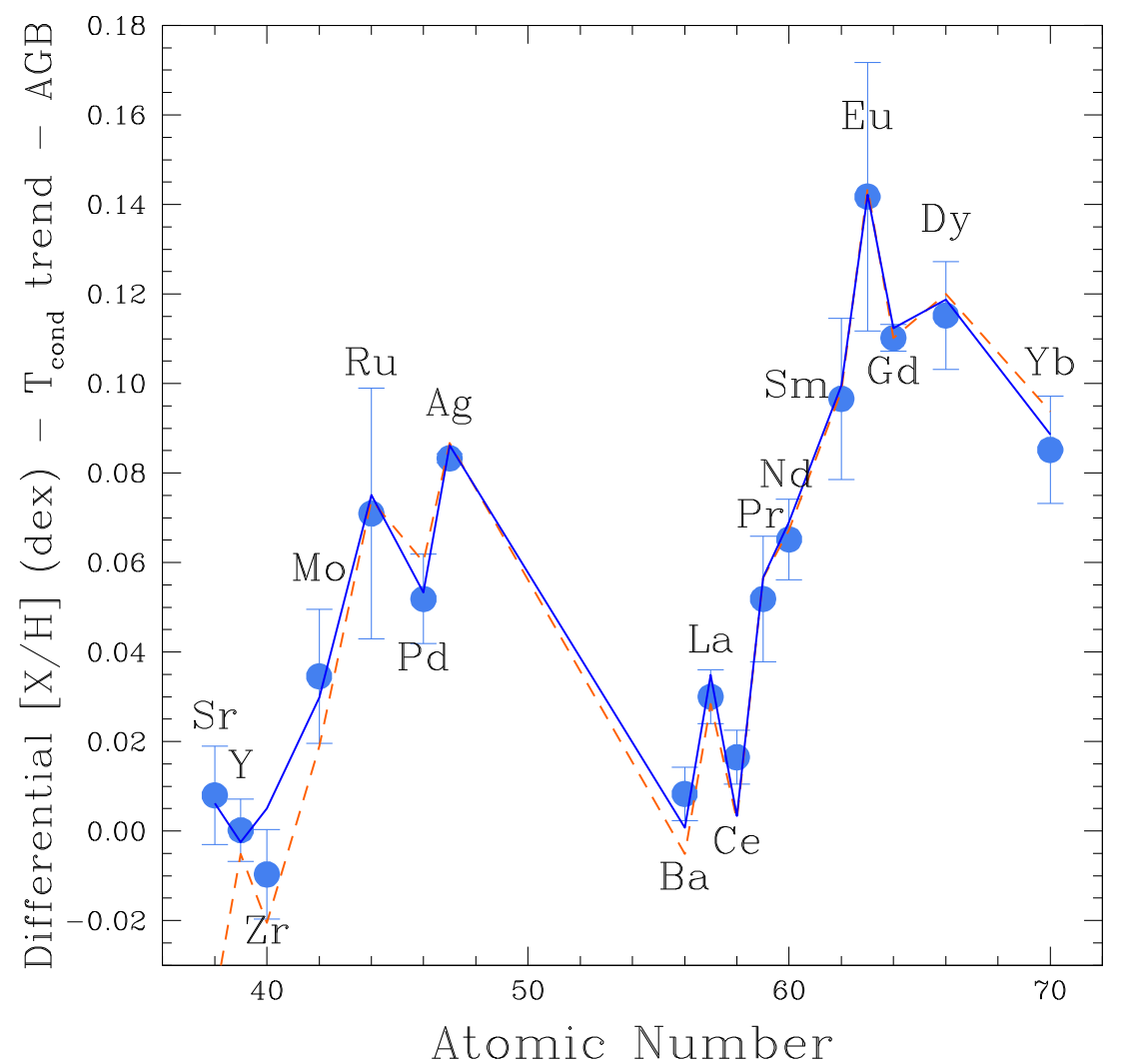

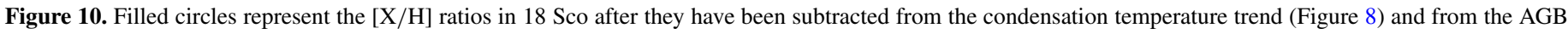

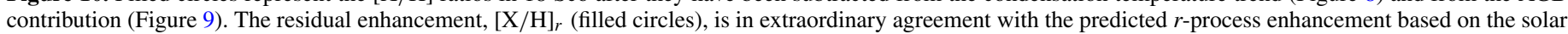
system $r$-process fractions by Simmerer et al. (2004) and Bisterzo et al. (2011, 2013), represented by dashed and solid lines, respectively.

(A color version of this figure is available in the online journal.)

and compare these results with the predicted enhancement based on the $r$-process fractions in the solar system, $r_{S S}=1-s_{S S}$, using the $s$ fractions $\left(s_{S S}\right)$ by Simmerer et al. (2004) and Bisterzo et al. (2011, updated for a few elements by Bisterzo et al. 2013). Then, we can estimate the $r$-process contribution $[\mathrm{X} / \mathrm{H}]_{r}^{S S}$ from

$$
1-s_{S S}=10^{[\mathrm{X} / \mathrm{H}]_{r}^{S S} / \Delta_{T}} / 10^{[\mathrm{X} / \mathrm{H}]_{T} / \Delta_{T}},
$$

where we define $\Delta_{T}$ as the average of the three most enhanced $s$-process and $r$-process elements, corresponding to $\Delta_{T}=$ $0.093 \mathrm{dex}$ for the observed $[\mathrm{X} / \mathrm{H}]_{T}$ enrichment in $18 \mathrm{Sco}$ (Table 4).

Therefore, the predicted $r$-process contribution based on the solar system $r$ fractions, $[\mathrm{X} / \mathrm{H}]_{r}^{S S}$, is

$$
[\mathrm{X} / \mathrm{H}]_{r}^{S S}=\Delta_{T} \times \log _{10}\left(1-s_{S S}\right)+[\mathrm{X} / \mathrm{H}]_{T} .
$$

In Figure 10, we compare the "observed" $r$-process enhancement $[\mathrm{X} / \mathrm{H}]_{r}$ (Equation (7)) with the predicted one based on the solar system $r$-process fractions, $[\mathrm{X} / \mathrm{H}]_{r}^{S S}$ (Equation (9)). There is an astonishing agreement, strongly suggesting that the remaining enhancement is indeed due to the $r$ process. The impressive agreement, even at the scale of the small fluctuations ( $\sim 0.02$ dex) among nearby n-capture elements (Figure 10), also suggests that even for heavy elements we succeeded in achieving abundances with errors of about 0.01 dex.

Another way to show that the residual enhancement (after subtracting the AGB contribution) is due to the $r$ process is by comparing the $r$ fractions of the $[\mathrm{X} / \mathrm{H}]_{T}$ ratios with the $r$ fractions in the solar system. Since the Bisterzo et al. $(2011,2013) s_{S S}$ fractions fit somewhat better our $[\mathrm{X} / \mathrm{H}]_{r}$ ratios
(Figure 10), we will use their values in the comparison. The "observed" $r$ fractions in the $[\mathrm{X} / \mathrm{H}]_{T}$ ratios in $18 \mathrm{Sco}$ are estimated by

$$
r=10^{[\mathrm{X} / \mathrm{H}]_{r} / \Delta_{T}} / 10^{[\mathrm{X} / \mathrm{H}]_{T} / \Delta_{T}} .
$$

The "observed" $r$ fractions and the solar system $r_{S S}$ fractions (Bisterzo et al. 2011, 2013) are compared in Figure 11. Again, the agreement is remarkable (slope of 0.90 and element-toelement scatter of only 0.04 ), showing that after both the $T_{\text {cond }}$ trend and the AGB contribution are subtracted, the remaining material can be explained by the $r$ process. Our high-precision abundances provide independent evidence of the universality of the $r$ process, i.e., of the remarkable similarity of the relative abundance pattern among different $r$-process elements. Our finding is similar to what is found in metal-poor stars, where the scaled solar $r$-process pattern matches the abundances of most neutron-capture elements (e.g., Cowan et al. 2002; Hill et al. 2002; Frebel et al. 2007; Sneden et al. 2008; Siqueira Mello et al. 2013).

The complex abundance pattern of 18 Sco can be thus explained by the condensation temperature trend, the $s$ process, and the $r$ process. The excess of refractory elements relative to the Sun seems to be typical of solar-like stars (Meléndez et al. 2009; Ramírez et al. 2009a, 2010; Schuler et al. 2011; Liu et al. 2014; Mack et al. 2014), so 18 Sco looks normal in this regard. As we will see below, the additional enhancement in the n-capture elements may be explained by the younger age of 18 Sco (Section 4).

A deficiency in the abundances of the $s$-process elements have been reported in our analysis of the pair of almost solar 


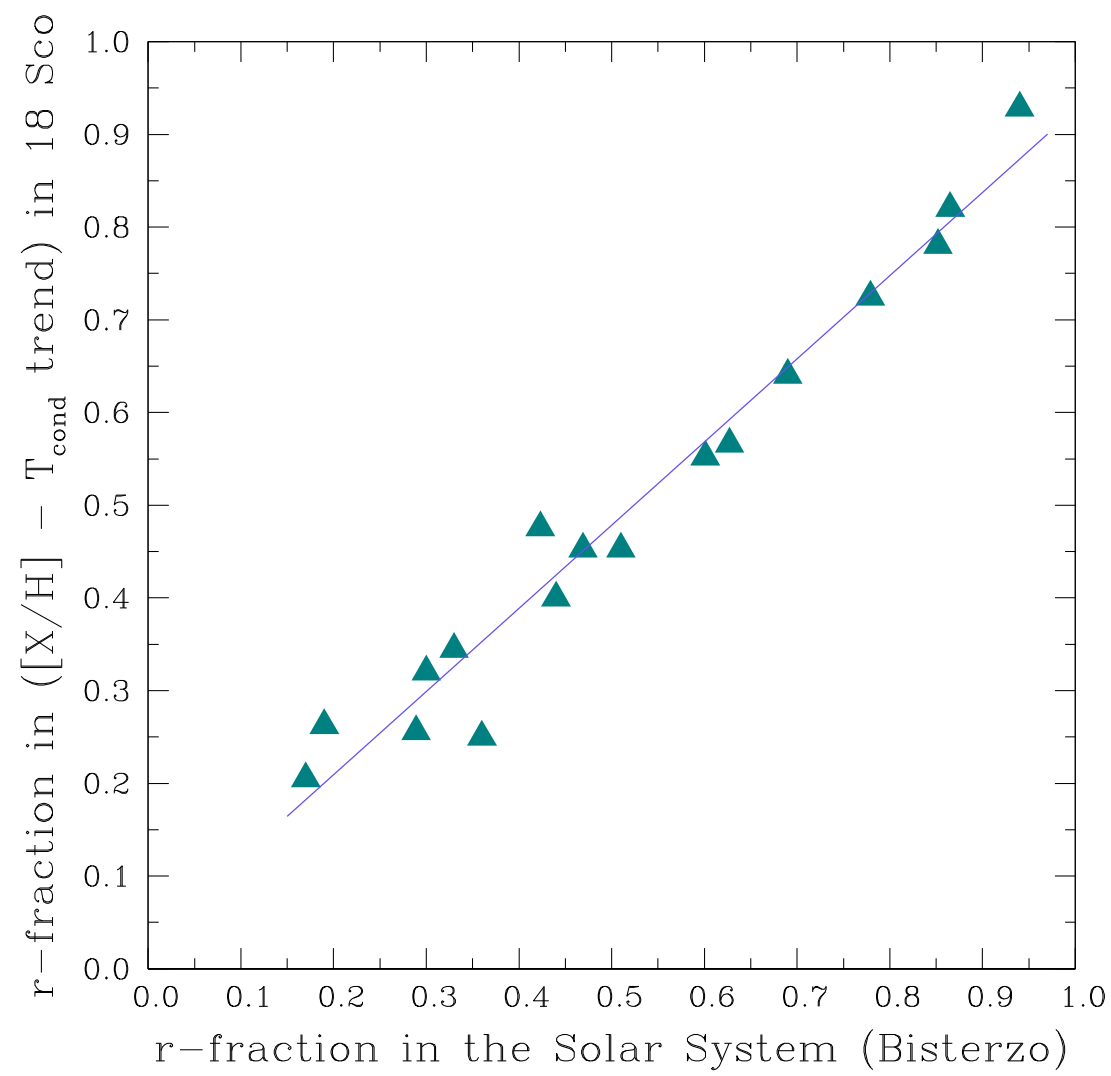

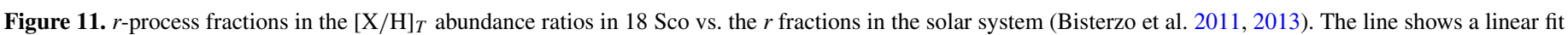
with slope of 0.90 and element-to-element scatter of only 0.04 .

(A color version of this figure is available in the online journal.)

twins 16 Cyg (Ramírez et al. 2011) and the solar twin HIP 102152 (Monroe et al. 2013), which are both older than the Sun by 2.5 and $3.7 \mathrm{Gyr}$, respectively. In contrast, the $s$-process elements seem enhanced in the young solar twin 18 Sco. Thus, the increasing enhancement of the $s$-process elements with decreasing age is probably due to the pollution of successive generations of AGB stars, which can be tracked using solar twins spanning ages from $2.9 \mathrm{Gyr}$ (18 Sco) up to $8.2 \mathrm{Gyr}$ (HIP 102152). Similar enhancements of the $s$-process elements have been found in open clusters (e.g., D’Orazi et al. 2009; Maiorca et al. 2011; Yong et al. 2012; Jacobson \& Friel 2013; Mishenina et al. 2013b), with Ba showing the clearest trend of increasing abundance for younger ages. A similar trend was observed in field stars (Edvardsson et al. 1993; Bensby et al. 2007; da Silva et al. 2012), but Mishenina et al. (2013a) found no dependence of $[\mathrm{Ba} / \mathrm{Fe}]$ and $[\mathrm{La} / \mathrm{Fe}]$ with age. Although there is no consensus yet, most evidence points to enhanced $s$-process abundance with decreasing age.

Regarding the $r$-process elements, da Silva et al. (2012) found a decrease of $[\mathrm{Sm} / \mathrm{Fe}]$ with decreasing age in stars younger than the Sun. Using both Eu abundances (4,129 $\AA$ line) and stellar ages determined by Bensby et al. (2005) in thin disk stars, we found that for ages lower than $9 \mathrm{Gyr}$ there is a flat $[\mathrm{Eu} / \mathrm{Fe}]$ ratio, i.e., no dependence with stellar age. Unfortunately, neither del Peloso et al. (2005) nor Mishenina et al. (2013a) studied the dependence of their $[\mathrm{Eu} / \mathrm{Fe}]$ ratios with age in thin disk stars. Our analysis of two old solar twins (Ramírez et al. 2011; Monroe et al. 2013) found normal $r$-process abundances for $16 \mathrm{Cyg} \mathrm{B}$ and HIP 102152. Based on the limited evidence, $r$-process elements seem to have a flat behavior with age.
Since 18 Sco is considerably younger than the Sun, the enhancement in the $s$-process elements could be due just to normal Galactic chemical evolution. However, the enhancement of the $r$-process elements in $18 \mathrm{Sco}$ is more difficult to understand because, based on our discussion above, those elements are not expected to be enriched.

The unexpected enhancement of the $r$-process elements in 18 Sco could be attributed to a somewhat higher contribution of $r$-process ejecta to the natal cloud of 18 Sco than around other solar-like stars.

Our precise abundances show that whatever sources produced the enrichment in the n-capture elements did not produce substantial quantities of elements lighter than $Z=30$. The amount of mass with $Z>30$ that would be needed to produce the observed enhancements is very small, only $2.7 \times 10^{-8} M_{\odot}$ and $2.4 \times 10^{-7} M_{\odot}$ for the $s$ and $r$ process, respectively.

\section{IMPLICATIONS FOR CHEMICAL TAGGING}

Studying the detailed abundance pattern of solar-type stars, we may be able to reconstruct the buildup of the Galaxy using chemical tagging (Freeman \& Bland-Hawthorn 2002). One of the main problems when tagging individual stars to a given natal cloud may be radial migration (Sellwood \& Binney 2002), yet the chemical abundances may be preserved, so by using chemical tagging stellar groups or clusters could be reconstructed.

Based on our precise abundances in $18 \mathrm{Sco}$, it is clear that different elements should be targeted for chemical tagging. First, as many as possible of the highly volatile $(\mathrm{C}, \mathrm{N}, \mathrm{O})$ and highly 
refractory elements $(\mathrm{Al}, \mathrm{Ti}, \mathrm{Sc}, \mathrm{Ca}, \mathrm{V}, \mathrm{Fe})$ should be analyzed to determine if there is any trend with condensation temperature. If possible it would be important to cover also some semivolatiles (e.g., S, Zn, Na, Cu, K, Mn, P) and some medium refractories (e.g., $\mathrm{Si}, \mathrm{Mg}, \mathrm{Cr}, \mathrm{Ni}, \mathrm{Co}, \mathrm{V}$ ), to verify if there is a break in the trend with condensation temperature (Meléndez et al. 2009). Also, it should be important not to discard stars from a given group due to small discrepant abundances because those anomalies could be due to either the formation of terrestrial (Meléndez et al. 2009) or giant (Tucci Maia et al. 2014; Ramírez et al. 2011) planets.

Some of the above elements are of course relevant to different nucleosynthetic processes such as production of $\alpha$ elements $(\mathrm{O}$, $\mathrm{S}, \mathrm{Ca}, \mathrm{Si}, \mathrm{Ti})$ by $\mathrm{SNe}$ II or signatures of proton-burning $(\mathrm{Na}$, $\mathrm{Mg}, \mathrm{Al}, \mathrm{O})$. Lithium is important as a potential chronometer (Baumann et al. 2010; Monroe et al. 2013; Melendez et al. 2014) and in the study of the transport mechanisms inside stars (e.g., Charbonnel \& Talon 2005; do Nascimento et al. 2009). The study of 18 Sco also shows the importance of including the heavy elements for chemical tagging. Ideally, at least some elements among $\mathrm{Sr}, \mathrm{Zr}$, Y, or Ba should be included to verify the $s$ process, and some elements among $\mathrm{Ru}, \mathrm{Pd}, \mathrm{Ag}, \mathrm{Sm}, \mathrm{Gd}$, $\mathrm{Eu}$, or Dy could be explored for the $r$ process.

\section{CONCLUSIONS}

We have performed the most precise and complete abundance analysis of 18 Sco. Being the brightest of the solar twins, very high $\mathrm{S} / \mathrm{N}$, high-resolution spectra were gathered for $18 \mathrm{Sco}$ and the Sun, and a strictly differential line-by-line analysis was performed, allowing us to achieve a precision of about $0.005 \mathrm{dex}$ in differential abundances. In addition, highly precise stellar parameters were obtained, which would be important for further modeling of this solar twin using different techniques. Precise radial velocities were gathered with HARPS, but no planet has been detected yet.

The complex abundance pattern of 18 Sco shows enhancements (relative to the Sun) in the refractory, $s$-process, and $r$-process elements. After subtracting the trend with condensation temperature and the contribution from AGB stars, the remaining enhancement shows the same pattern as the $r$ process in the solar system. This shows the universality of the $r$ process. The different contributions to the abundance enrichment in 18 Sco could be disentangled thanks to the exquisite precision achieved in our work.

The 18 Sco serves as a test bed for studies of chemical tagging in large samples of stars of upcoming surveys, such as HERMES, ${ }^{15}$ which plans to survey about a million stars at high spectral resolution.

J.M. and T.R.M. acknowledge support from FAPESP (2012/ 24392-2 and 2010/19810-4). M.B. and J.B. acknowledge support for this work from the NSF (grant No. AST-1313119)

Facilities: VLT (UVES), Keck (HIRES), ESO:3.6m (HARPS)

\section{REFERENCES}

Arlandini, C., Käppeler, F., Wisshak, K., et al. 1999, ApJ, 525, 886 Asplund, M., Grevesse, N., Sauval, A. J., \& Scott, P. 2009, ARA\&A, 47, 481 Barnes, S. A. 2007, ApJ, 669, 1167

Baumann, P., Ramírez, I., Meléndez, J., Asplund, M., \& Lind, K. 2010, A\&A, 519, A 87

\footnotetext{
15 http://www.aao.gov.au/science/instrumentation/hermes/
}

Bazot, M., Ireland, M. J., Huber, D., et al. 2011, A\&A, 526, L4

Bazot, M., Campante, T. L., Chaplin, W. J., et al. 2012, A\&A, 544, A106

Bensby, T., Feltzing, S., Lundström, I., \& Ilyin, I. 2005, A\&A, 433, 185

Bensby, T., Zenn, A. R., Oey, M. S., \& Feltzing, S. 2007, ApJL, 663, L13

Bergemann, M., \& Cescutti, G. 2010, A\&A, 522, A9

Bergemann, M., \& Gehren, T. 2008, A\&A, 492, 823

Bergemann, M., Lind, K., Collet, R., Magic, Z., \& Asplund, M. 2012, MNRAS, 427, 27

Bisterzo, S., Gallino, R., Straniero, O., Cristallo, S., \& Käppeler, F. 2011, MNRAS, 418, 284

Bisterzo, S., Travaglio, C., Wiescher, M., et al. 2013, arXiv:1311.5381

Boyajian, T. S., McAlister, H. A., van Belle, G., et al. 2012, ApJ, 746, 101

Bressan, A., Marigo, P., Girardi, L., et al. 2012, MNRAS, 427, 127

Bruning, D. H. 1984, ApJ, 281, 830

Castelli, F., \& Kurucz, R. L. 2004, arXiv:astro-ph/0405087

Cayrel de Strobel, G. 1996, A\&ARv, 7, 243

Chanamé, J., \& Ramírez, I. 2012, ApJ, 746, 102

Charbonnel, C., \& Talon, S. 2005, Sci, 309, 2189

Cowan, J. J., Sneden, C., Burles, S., et al. 2002, ApJ, 572, 861

Cox, A. N. 2000, Allen's Astrophysical Quantities (4th ed.; New York: AIP Press/Springer)

da Silva, R., Porto de Mello, G. F., Milone, A. C., et al. 2012, A\&A, 542, A84

Datson, J., Flynn, C., \& Portinari, L. 2012, MNRAS, 426, 484

Datson, J., Flynn, C., \& Portinari, L. 2014, MNRAS, 439, 1028

del Peloso, E. F., Cunha, K., da Silva, L., \& Porto de Mello, G. F. 2005, A\&A, 441, 1149

Demarque, P., Woo, J.-H., Kim, Y.-C., \& Yi, S. K. 2004, ApJS, 155, 667

Denissenkov, P. A. 2010, ApJ, 719, 28

Do Nascimento, J. D., Jr., Castro , M., Meléndez, J., et al. 2009, A\&A, 501, 687

D’Orazi, V., Magrini, L., Randich, S., et al. 2009, ApJL, 693, L31

Edvardsson, B., Andersen, J., Gustafsson, B., et al. 1993, A\&A, 275, 101

Frebel, A., Christlieb, N., Norris, J. E., et al. 2007, ApJL, 660, L117

Freeman, K., \& Bland-Hawthorn, J. 2002, ARA\&A, 40, 487

González Hernández, J. I., Israelian, G., Santos, N. C., et al. 2010, ApJ, 720,1592

Hall, J. C., Henry, G. W., \& Lockwood, G. W. 2007, AJ, 133, 2206

Hill, V., Plez, B., Cayrel, R., et al. 2002, A\&A, 387, 560

Jacobson, H. R., \& Friel, E. D. 2013, AJ, 145, 107

Karakas, A. I. 2010, MNRAS, 403, 1413

Kim, Y.-C., Demarque, P., Yi, S. K., \& Alexander, D. R. 2002, ApJS, 143, 499 Li, T. D., Bi, S. L., Liu, K., Tian, Z. J., \& Shuai, G. Z. 2012, A\&A, 546, A83

Lind, K., Asplund, M., \& Barklem, P. S. 2009, A\&A, 503, 541

Liu, F., Asplund, M., Ramírez, I., Yong, D., \& Meléndez, J. 2014, MNRAS, 442, L51

Lodders, K. 2003, ApJ, 591, 1220

Luck, R. E., \& Heiter, U. 2005, AJ, 129, 1063

Mack, C. E., III, Schuler, S. C., Stassun, K. G., \& Norris, J. 2014, ApJ, 787, 98

Maiorca, E., Randich, S., Busso, M., Magrini, L., \& Palmerini, S. 2011, ApJ, 736, 120

Meléndez, J., Asplund, M., Gustafsson, B., \& Yong, D. 2009, ApJL, 704, L66

Meléndez, J., Bergemann, M., Cohen, J. G., et al. 2012, A\&A, 543, A29

Meléndez, J., \& Ramírez, I. 2007, ApJL, 669, L89

Melendez, J., Schirbel, L., Monroe, T. R., et al. 2014, A\&A, 567, L3

Mishenina, T. V., Pignatari, M., Korotin, S. A., et al. 2013a, A\&A, 552, A128

Mishenina, T., Korotin, S., Carraro, G., Kovtyukh, V. V., \& Yegorova, I. A. 2013b, MNRAS, 433, 1436

Monroe, T. R., Meléndez, J., Ramírez, I., et al. 2013, ApJL, 774, L32

Neves, V., Santos, N. C., Sousa, S. G., Correia, A. C. M., \& Israelian, G. 2009, A\&A, 497, 563

Petit, P., Dintrans, B., Solanki, S. K., et al. 2008, MNRAS, 388, 80

Porto de Mello, G. F., \& da Silva, L. 1997, ApJL, 482, L89

Porto de Mello, G. F., da Silva, R., da Silva, L., \& de Nader, R. V. 2014, A\&A, 563, A52

Ramírez, I., Allende Prieto, C., Lambert, D. L., Koesterke, L., \& Asplund, M. 2009b, MmSAI, 80, 618

Ramírez, I., Asplund, M., Baumann, P., Meléndez, J., \& Bensby, T. 2010, A\&A, 521, A33

Ramírez, I., Meléndez, J., \& Asplund, M. 2009a, A\&A, 508, L17

Ramírez, I., Meléndez, J., \& Asplund, M. 2014a, A\&A, 561, A7

Ramírez, I., Meléndez, J., Cornejo, D., Roederer, I. U., \& Fish, J. R. 2011, ApJ, 740,76

Ramírez, I., Michel, R., Sefako, R., et al. 2012, ApJ, 752, 5

Ramírez, I., et al. 2014b, A\&A, submitted

Saar, S. H., \& Osten, R. A. 1997, MNRAS, 284, 803

Schuler, S. C., Flateau, D., Cunha, K., et al. 2011, ApJ, 732, 55

Sellwood, J. A., \& Binney, J. J. 2002, MNRAS, 336, 785

Simmerer, J., Sneden, C., Cowan, J. J., et al. 2004, ApJ, 617, 1091 
Siqueira Mello, C., Spite, M., Barbuy, B., et al. 2013, A\&A, 550, A122

Sneden, C. A. 1973, PhD thesis, Univ. of Texas at Austin

Sneden, C., Cowan, J. J., \& Gallino, R. 2008, ARA\&A, 46, 241

Soubiran, C., \& Triaud, A. 2004, A\&A, 418, 1089

Sousa, S. G., Santos, N. C., Israelian, G., Mayor, M., \& Monteiro, M. J. P. F. G. 2007, A\&A, 469, 783

Sousa, S. G., Santos, N. C., Mayor, M., et al. 2008, A\&A, 487, 373

Takeda, Y., Kawanomoto, S., Honda, S., Ando, H., \& Sakurai, T. 2007, A\&A, 468,663
Takeda, Y., \& Tajitsu, A. 2009, PASJ, 61, 471

Tanner, A. M., Gelino, C. R., \& Law, N. M. 2010, PASP, 122, 1195

Trilling, D. E., Bryden, G., Beichman, C. A., et al. 2008, ApJ, 674, 1086

Tsantaki, M., Sousa, S. G., Adibekyan, V. Z., et al. 2013, A\&A, 555, A150

Tucci Maia, M., Meléndez, J., \& Ramírez, I. 2014, ApJ, in press

Xiong, D. R., \& Deng, L. 2009, MNRAS, 395, 2013

Yi, S., Demarque, P., Kim, Y.-C., et al. 2001, ApJS, 136, 417

Yong, D., Carney, B. W., \& Friel, E. D. 2012, AJ, 144, 95 